\title{
PHOTOGRAMMETRISCHES FELSZEICHNEN
}

\author{
Willi Kreisel
}

\section{GES CH I CH T L I CHES}

Der Photogrammeter-Geodät ${ }^{1}$ brachte als Geschenk an die Topographie die geumetrisch absolut genaue Höhenlinie im Felsgebiet. Um die Bedeutung dieses neuen Darstellungsmittels richtig einschätzen zu können, sei zunächst das Auftauchen der Höhenkurve in der Kartographie überhaupt, also als Höhenlinie in nichtfelsigem Gebiet, beleuchtet. Der Vergleich ergibt nämlich auffallende Übereinstimmung mit der Verwendung der Höhenlinie als Felskurve.

Ums Jahr 1800 tauchen in der Geländedarstellung mit Vertikalprojektion die ersten Schraffenkarten auf. Die Felszeichnung erfolgte ebenfalls mit Schraffen. Obschon die geometrische Lage so wie die Dicke der Schraffen bald normiert wurde, indem man die Schraffe entweder in der Fall-Linie oder horizontal verlaufen ließ, blieb sie im Felsgebiet wild, d. h. auch die schräge Schraffe blieb zulässig. Immerhin wahrte man die Einheitlichkeit im Kartenbild insofern, als man für Fels und übriges Terrain die Schraffe als Darstellungsmittel beließ.

Nach 1800 lernte man dann langsam auch die Terrainkurve aufzunehmen, und ebenso zögernd fand schließlich dieses neue Darstellungsmittel Eingang in die reproduzierte Karte. Dies geschah allerdings nicht kampflos. Prominente Kartenbenützer legten sofort klar, daß die Kurvenmethode die physische Darstellung des Geländes allzusehr vernachlässige. Der Instrumentenbau hatte den Fortschritt bedingt, und der Techniker glaubte, fälschlicherweise, das Darstellungsproblem endgültig neu gelöst zu haben.

Versuche, dem Höhenkurvenbild markante morphologisch begründete Terrainlinien beizufügen und so zu einer besseren physischen Darstellung zu kommen, blieben mit Recht aus. Solche Terrainlinien sind in der Regel im Gelände zu wenig scharf ausgeprägt. Ihre endgültige Fixierung ist Aufgabe der Geomorphologie.

Wo solche Terrainlinien im Terrain deutlich erkennbar sind wie bei Gräten, Talwegen, Abrissen, Kanten usw., werden sie in der Regel durch die üblichen topographischen Signaturen oder durch das Kurvensystem genügend scharf erfaßt. In der klassischen Periode der schweizerischen Topographie war es zudem immer üblich, prägnante morphologische Kleinformen zusätzlich zu den Kurven mit Schraffen auszumodellieren. Man sehe sich einmal das Blatt Gemmi 1:50000 des Siegfriedatlasses an, das Becker 1881/82 neu aufgenommen hat. Was da an Terrassenrändern, Bachbetterrassen, Abrissen, Schlipfen, Moränen (auch im bewachsenen Gebiet), Bergsturzhügeln, Rundhöckern usw. mit Schraffen zusätzlich zu den Kurven aufgenommen wurde, ist erstaunlich (Abb. 1). Leider tilgte die Photogrammetrie diese alte Topographen-Gepflogenheit aus und der moderne Photogrammeter-Topograph plagt sich mit ihnen kaum mehr ab. Die alten Bach-Terrassenränder und Moränen im Boden des Engstligenalp-Kares findet man nur in der alten Aufnahme von Becker (Abb. 1), auf der neuen Landeskarte (Blatt Wildstrubel) dagegen nicht mehr; ebensowenig die Moränenreste und Rundhöcker am Ausgang des Karbodens. Dasselbe konstatieren wir beim Ausgang des Trubelnkares auf der Südseite des Gemmipasses. Die neue Landeskarte beschränkt sich auf die Kurven und deren engere Äquidistanz von 20 anstatt

1 Die Photogrammetrie ist eine geodätische Wissenschaft mit topographischem Thema. Sie wird hauptsächlich von Geodäten betrieben. Dabei blieben aber etliche Forderungen der Topographie unerfüllt. Es hat sich deshalb im Laufe der Jahre eine Kompromißlösung herauskristallisiert, indem die schon in der napoleonischen Topographie übliche $Z_{w e i t e i l u n g}$ in analytische und synthetische Topographie, wie ich es nenne, einerseits den Photogrammeter-Geodäten, anderseits den Photogrammeter-Topographen entstehen ließ. Der Verfasser gehört als alter Gebirgstopograph zu der zweiten Kategorie. 

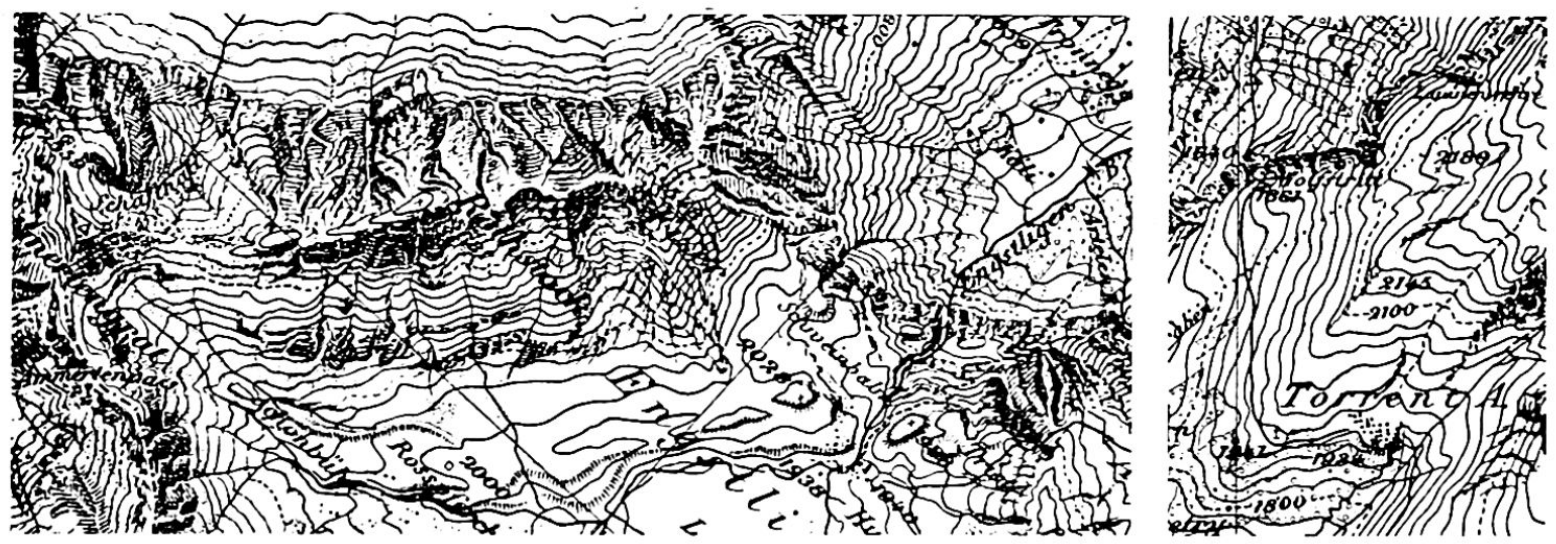

Abb. 1 Zwei Ausschnitte aus Blatt Gemmi SA 473. Aufnahme Becker 1881-82. Herausmodellierung von Kleinformen mit Schraffen. 1:1

$30 \mathrm{~m}$. Das Resultat befriedigt aber nicht. Schon die bloße Betrachtung entscheidet für Becker, die Feldbenützung erst recht. Ganz glcich bei den Bergsturzhügeln auf der Spittelmatte am Gemmiweg und im Kandergrund (Blatt Adelboden, SA). Und welch hübsches, klares Bild zeigt die Torrentalp südlich des Gemmipasses mit dem schraffierten Terrassenrand bei Becker (Abb. 1) gegenüber der Landeskarte!

Aber nicht nur Kleinformen, sondern auch größere Gebilde hat der alte Topograph nach Schweizermanier explizite herausgearbeitet. Diese Fazettierung der größeren Formen erfolgte vornehmlich nach geometrischen Formen, die notgedrungen meistens mit morphologischen Flächen identisch waren. Dies geschah aber blo $\beta$ in Bleistift auf dem Meßtischblatt. In der ausgearbeiteten Aufnahme verschwanden diese Fazetten wieder, und mit Recht, denn es waren bloß Hilfslinien zur Konstruktion des Kurvenbildes. Erst der geschulte Geomorphologe kann, wie bereits oben schon angedeutet wurde, solche Linien mit Garantie herausschälen; sein Produkt gehört dann aber in die morphologische und nicht in die topographische Karte.

Die ersten positiven Versuche, das physische Manko der Terrainkurven (fehlende Gesamterfassung des Terrains) zu beheben, bestanden in der Kombination der Schraffe mit dem neuen Darstellungsmittel der Höhenkurve. Man druckte Kurven über Schraffen und zwar in verschiedensten Kombinationen. Doch brachte keiner dieser vermittelnden Versuche befriedigende Resultate.

Eine andere Lösung bestand darin, daß man Doppelausgaben derselben Karte publizierte, die eine Ausgabe in Kurven, die andere in Schraffen. Auch solche Lösungen sind vollständig aus der Produktion verschwunden.

Die endgültige Abhilfe gegen den technischen Irrtum, daß die Höhenkurve alles Nötige leisten könne, kam langsam in Form eines modellierten Terraintones, der das ganze Kartenbild erfaßte. Zwar konnte man auch beim Kupferstich mit Roulette oder Sandkornätzung modellierte Töne erzeugen. Doch wurden diese Methoden nie ergiebig angewendet. Anders bei der etwa 1830 aufkommenden Lithographie. Sie gestattete, die gesamte Terrainfläche wesentlich leichter mit einem modellierten Ton vermittelst einer Schummerung zu erfassen. Da Kurven und Schummerung zwei Darstellungsmittel sind, die sich, im Gegensatz zu Kurve und Schraffe, im Zusammendruck gut vertragen, ergab diese Methode vollen Erfolg. Dabei gewannen die erwähnten Terrainlinien beträchtliche Bedeutung. Der Kartograph, der die Terrainoberfläche als physische Fläche gesamthaft mit Schummerung versah, könnte diese Terrainlinien oder gar die Fazetten sehr gut gebrauchen, besonders wenn es garantierte morphologische Fazetten sind. Dies beweisen Skizzen zu meinem im Entstehen begriffenen Kar-Atlas. Es zeigte sich aber, daß man davon nicht allzu viel erwarten darf, denn in der Natur sind die reinen Formtypen nicht so häufig, zudem sind sie vielfach verwischt, überlagert und kombiniert, wodurch ausgesprochen morphologische 


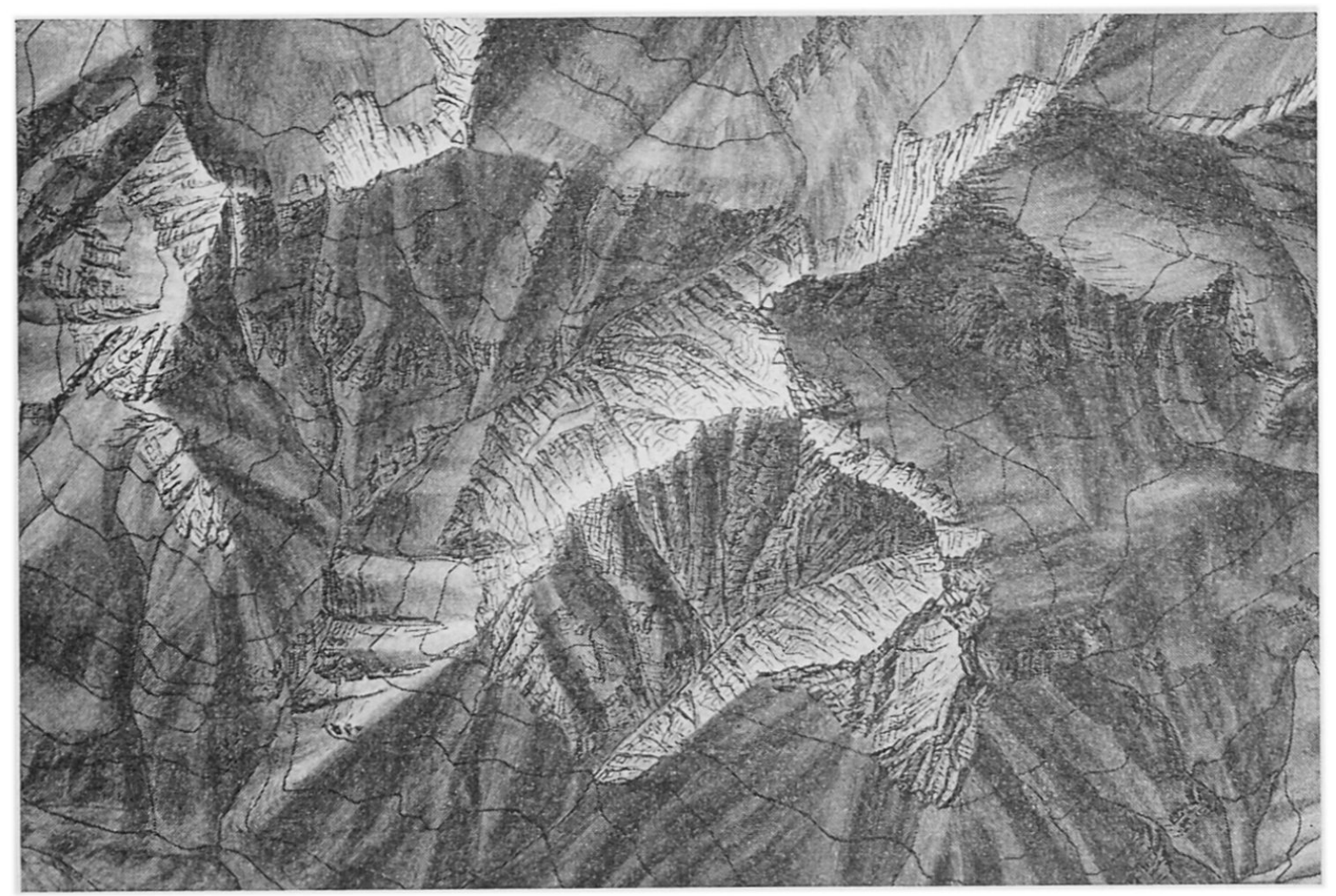

Abb. 2 Ausschnitt aus einer Kartenskizze, in welcher der Reliefton in schiefer Beleuchtung über eine offene Felsschraffen-Zeichnung gelegt ist. Zeichnung und Bemalung vom Verfasser auf Grund des französischen 20000 stels (Westalpen). Verkleinert (zu stark)

Linien nicht so oft erkennbar sind. Die topographisch verwendbaren Resultate der Morphologie bilden einen verwunderlich kleinen Teil der Fläche auf einem Meßtischblatt. Selbstverständlich hat jeder Quadratzentimeter auf dem Blatt seine morphologische Erkennungsmarke. Der Topograph aber braucht sich nicht um alle zu kümmern. Da die morphologischen Aufnahmen in der Regel später erfolgen als die topographischen, muß sich der Kartograph ohnehin meistens mit eigenen Mitteln helfen. Logisch und interessant wäre natürlich, daß man heute (im Zeitalter der Photogrammetrie) diese morphologische Fazettierung bereits am Autographen vornähme, ja sogar schon auf dem kopierten Fliegerbild. Das ergäbe für den Photogrammeter ein zielbewußtes Auswerten am Stereoskop und die besten Grundlagen zum Generalisieren. Es wäre das das photogrammetrische Analogon zur früheren Meßtisch-Fazettierung. Organisatorisch-betriebswirtschaftlich wird dies kaum je möglich sein. Es gäbe aber Bilder in der Linie der Beckerschen Meßtisch-Aufnahmen, bloß entsprechend genauer.

In Felsgebieten wurde indes weiterschraffiert, denn mit der Meßtischtopographie konnte man im Fels keine Kurven aufnehmen. Die Lösung, den Schummerton auch über die schraffierten Felsen zu legen, befriedigte nicht. Legt man die Felsschraffen sehr offen, so geht es schon etwas besser (Abb. 2 und 3). Das Aufkommen der Felskurven zeitigte nun im Felsbild genau die gleiche Entwicklung mit den analogen Ergebnissen.

Zuerst versuchte man die vermittelnden Lösungen: Kombination von Schraffen und Kurven im Fels. Jedoch kaum eine befriedigte, denn die wilde Felsschraffe paßt noch schlechter zur Höhenkurve als die normierte Geländeschraffe. Mich persönlich befriedigt von den vermittelnden Lösungen, und dies auch bloß für Spezialfälle, lediglich folgende: Dunkelbraune Felskurven mit Schraffenbild in weicher Bleistiftzeichnung. Sie ergeben eine verblüffend schöne Darstellung. Weiche Bleistiftzeichnung 


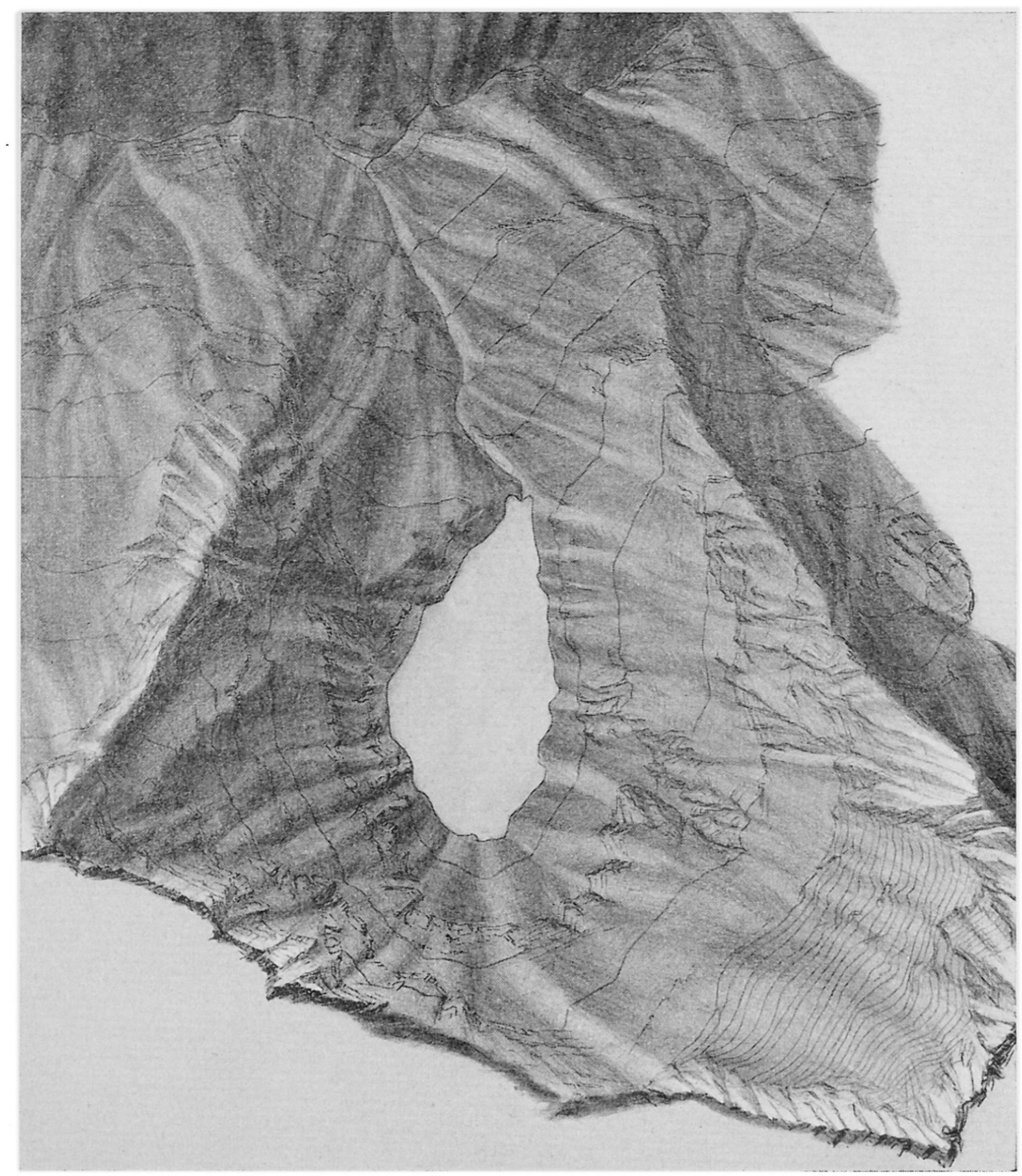

Abb. 3 Ausschnitt aus dem Übersichtsplan Chironico. Laghetto-Trog am Übergang aus der Val Chironico (Val Leventina, Tessin) in die Val Verzasca (Sonogno). Offene Felsschraffenzeichnung. Reliefton in schiefer Beleuchtung über die ganze Skizze. Zeichnung und Bemalung vom Verfasser. Verkleinert

wirkt absolut genügend scharf, sie wirkt sogar besser als eine mit hartem Stift gezeichnete und läßt braune Kurven noch genügend durchscheinen. Diese Lösung ist auch drucktechnisch, nicht nur in Originalzeichnung, durchführbar. Rationell ist dabei, die Kurven zum vorneherein braun zu drucken und die Felsschraffen von Hand mit Bleistift anzubringen (Abb. 4). Muß man auch die Schraffen drucken, so wird man die Schraffenvorlage entsprechend bearbeiten. Im großen und ganzen aber haben alle die vermittelnden Lösungen versagt, wo sie noch gehandhabt werden, sollten sie baldmöglichst aus der Produktion verschwinden.

Dasselbe gilt für die Doppelausgaben. Alles drängte viel eher auf eine neue Lösung. Sie bestand wie bei der Terrainkurve im Hinzufügen einer Schummerung zur 


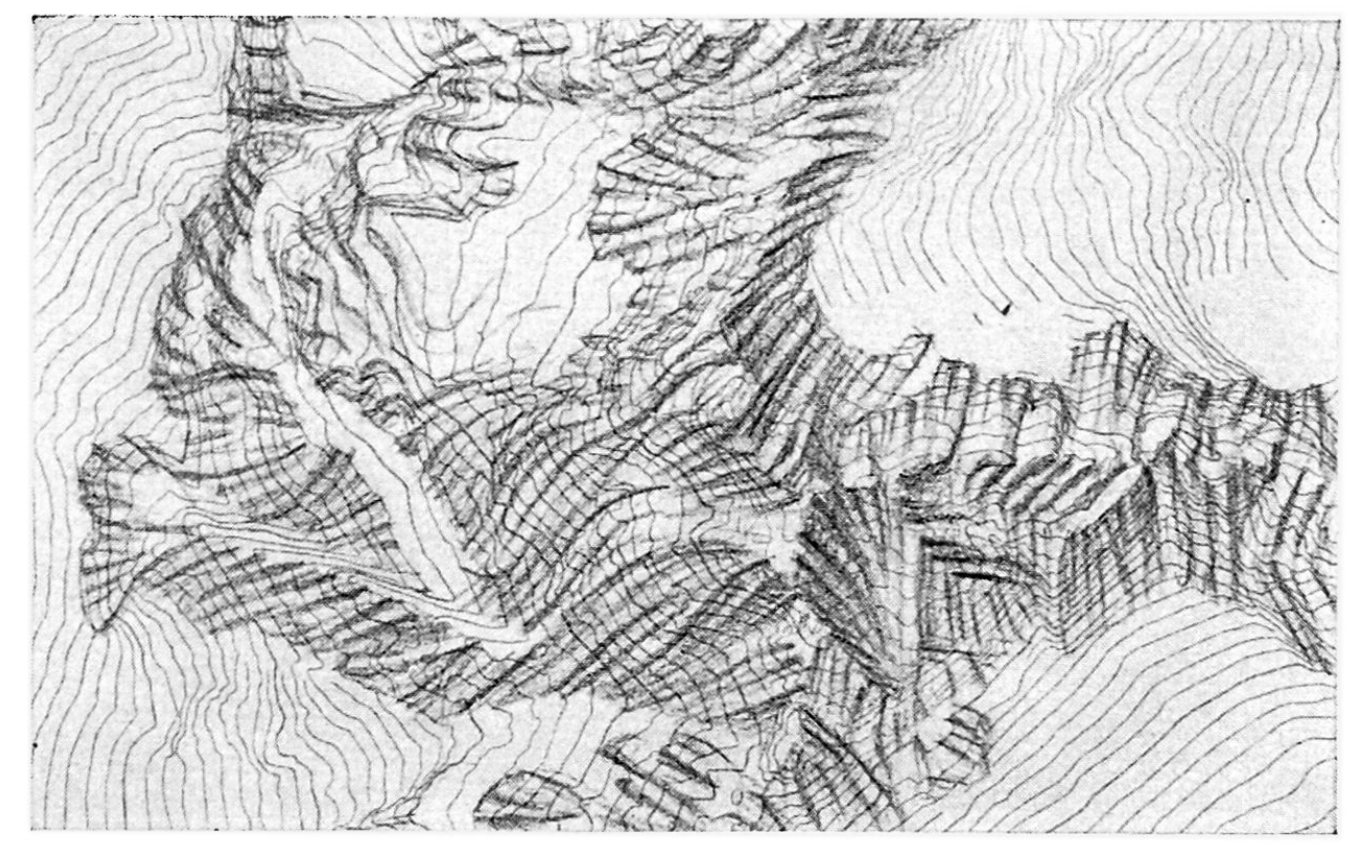

Abb. 4 Vermittelnde Methode. Felskurven und Felsschraffen, letztere in Bleistift. Mürtschenstock. Zeichnung vom Verfasser. 1:1

Felskurve. Während aber bei der Terrainkurve die Schummerung genügte, um zusammen mit der Terrainkurve die physische Form zu erfassen, und beide zusammen so dem Akzentuierungsgrad des Terrains fast vollkommen gewachsen waren, galt dies für die Kombination von Felskurve und Felsschummerung nicht. Erst die Hinzufügung eines weiteren Darstellungsmittels, nämlich der Felslinie, rettete im Felsbild die Situation. Im Felsgebiet sind die Fazettenlinien, die wir bereits als Geländelinien kennenlernten, sogar dazu bestimmt, die führende Rolle in der definitiven Felsdarstellung zu übernehmen. Wir nennen sie im Felsgebiet nicht mehr Geländelinien, sondern Felslinien ${ }^{2}$.

Zusammenfassend sei wiederholt: Die Entwicklung der Instrumententechnik gestattete anfangs bloß Nichtfelskurven aufzunehmen; im Felsgebiet blieb die Schraffe bestehen. Erst der Photogranımeter war infolge seiner neuen instrumentellen Ausrüstung imstande, Felskurven zu zeichnen; die Felsschraffe aber blieb sonderbarerweise im fertigen Kartenbild bestehen. Man wertete lange Zeit noch Felskurven aus und überdeckte die Kurven mit Schraffen. Die Ratlosigkeit gegenüber den neuen graphischen Möglichkeiten war beinahe total! Der bedeutendste Pionier auf dem Boden der schweiz. praktizierenden Photogrammetrie, R. Helbling, Flums, äußerte sich 1921 zwar noch recht vorsichtig und tolerant:

«Folgerichtig wäre es nun, nachdem die Autogrammetrie das Mittel ist, mit Leichtigkeit auch Felsgebiete in Horizontalkurven wiederzugeben, solche auch allgemein, also auch für

2 Der Verfasser hat als junger Ingenieur der Eidg. Landestopographie alle möglichen Kombinationen von Felsschraffe und Felskurve in Zeichnung und Druck durchexerziert. Die Landestopographie hat das Problem dann unter den Direktionen der Herren K. SchneIDER und neuerdings Prof. S. Bertschmann, weiterhin aufmerksam und durch weitere Versuche verfolgt. Sie ist sich sicher über alle Lösungen völlig klar. Für den neuen, bereits in Publikation befindlichen Gebirgs - 25 ooostel verwandte sie noch eine vermittelnde Lösung. Sie kündigt aber bereits für das Blatt Glärnisch eine neue Lösung des Problemes an. Es ist nicht daran zu zweifeln, da $\beta$ bei den materiellen und personellen Mitteln, die sie besitzt, diese neue Lösung befriedigen wird. Ähnlich liegen die Verhältnisse bei der Eidg. Vermessungsdirektion, welche für die Grundbuchpläne und deren Übersichtspläne besorgt ist. Noch sind dort Felskurven und eine stark stilisierte Felsliniensignatur gebräuchlich. Es ist aber, wie mir Herr Direktor HAERRY mitteilte, die Bahn frei für jede vernünftige Weiterentwicklung des Problems. Für eine neue, nicht vermittelnde Lösung ist ferner von Anfang an mit Schreib- und Zeichnungsfeder Ing. BLUMER in Bern eingetreten. (Siehe u. a. seine Glärnischkarte 1:25 000.) 

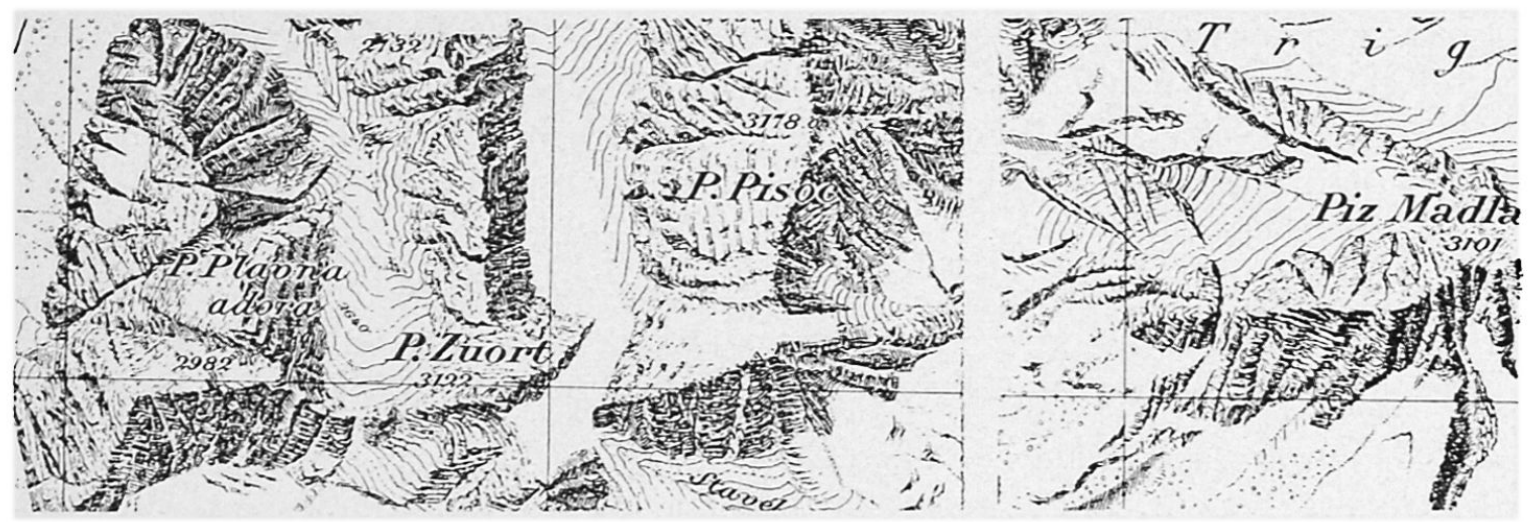

Abb. 5 Zwei Ausschnitte aus Blatt Scarl SA 425. Aufnahme Stergel 1848-49. Schwarzplatte. Felsschraffenzeichnung. STENGEL war zweifellos der begabteste Topograph der Dufourperiode. Er ist aber nicht bloß Topograph, sondern auch ein versierter Kartograph. Es ist derselbe STENGel, der auch die ersten Beispiele von mehrfarbigen Reliefkarten gemalt hat. Er ist damit ein Phänomen der Dufourperiode, ähnlich wie IMFELD und BECKER in der spätern Siegfriedperiode topographische Phänomene waren. $1: 1$

die Siegfriedkarte zu verlangen. Aber diese Forderung wäre zum mindesten verfrüht. Eine Karte, also eine Geländewiedergabe in kleinem Maßstabe bis zu etwa 1:20 0oo, soll vor allem einen raschen Überblick und eine rasche Orientierung im Gelände selber ermöglichen; das gilt für militärischen Gebrauch, für den Bergsteiger und Wanderer, den Techniker und Wissenschaftler, kurz für die Mehrzahl der Gebraucher, und wir müssen daran festhalten, daß die Methode der Siegfriedkarte diesen Zweck in bis heute unerreicht praktischer Weise erfüllt, während eine reine Kurvenzeichnung diesen Anforderungen, auch für einen geübten Kartenleser, nicht entspricht. Damit soll nicht gesagt sein, $\mathrm{da} B$ forschender und künstlerischer Geist nicht doch eine Lösung des Problems der Felsdarstellung finden könne, die näher am geometrischen Ideal liegt als die bisherige Schraffenzeichnung.» (Die stereoautogrammetrische $\mathrm{Ge}$ ländevermessung Schw. Bauztg. 77, 1921, 5, 38.)

Wenn das photogrammetrische Felsproblem gelöst werden soll, wird eine neue Konzeption nur zu finden sein auf der Basis großer praktischer Erfahrung in der alten Schraffenmethode und vieler Arbeit am photogrammetrischen Auswertegerät. Die Widerstände aber überwindet man schließlich bloß, indem man die Sache analytisch anpackt. Hier sei daher vorerst eine Analyse der alten Felsschraffenmethode gegeben, aber nur soweit, als sie für die neue photogrammetrische Methode wichtig ist.

\section{DIE HERKÖMMLICHE SCHRAFFENMETHODE}

Die geometrische Form. Durch Vorwärtseinschneiden bestimmt der Topograph in steilem Felsgebiet die ihm nötig erscheinende Anzahl von Punkten. Dieses Punktnetz versieht er laufend, so wie es entsteht, mit einer leichten Gerippezeichnung. Es handelt sich um die früher erwähnte Fazettierung des Felsgeländes vermittelst Felslinien, wie ich sie genannt habe.

Die physische Form ${ }^{3}$. Ebenfalls im Feld, am Meßtisch, angesichts der Natur wird die geometrische Grundlage der Felslinien zur Abbildung der physischen Form körperlich ausgestaltet. Dies geschieht durch Schraffierung mit Bleistift und ist nichts anderes als ein Landschaftszeichnen mit den Fesseln der geometrisch bestimmten Punkte. Statt der Perspektive gelten aber die Gesetze der parallelen Vertikalprojektion. Die Kunst der Auswahl und der Darstellung ist mit derjenigen des Landschaftszeichners identisch. Die Bleistiftzeichnung wird dann im Büro mit Feder und schwarzer Tusche überarbeitet. An einer guten Bleistiftzeichnung wird dabei kaum mehr etwas geändert. Tusch- und Bleistiftgummi gehören weder während noch nach ab-

3 Die Unterscheidung geometrische und physische Form wurde von dem napoleonischen Ingenieur-Geographen PUISSANT in die französische Topographie eingeführt. Sie ist kaum streng logisch, aber praktisch und so gemeint, daß erst die Schraffen die physische Form vollständig erfassen. 
geschlossener Überzeichnung auf den Arbeitstisch des gewandten Topographen; höchstens darf da und dort mit dem Schaber ein Strich verbessert werden.

Eine Analyse der Schraffentechnik lehrt, daß dieselbe bei der Erzeugung der physischen Form wiederum aus verschiedenen Komponenten besteht:

a) Aus der Auffassung der physischen Form. Beim Verarbeiten von bestehenden Felsdarstellungen fällt sofort auf, daß die Darstellungstechnik stark variiert. Um sich bei solchen Analysen kurz ausdrücken zu können, hat der Verfasser bereits 1925 in Bezug auf die Auffassung folgende zwei Bezeichnungen eingeführt.

1. Die orographische Darstellung (Oros $=$ Gebirge). Die Aufgabe wird lediglich geometrisch aufgefaßt. Der Topograph sieht am Matterhorn z. B. hauptsächlich den Vierkanter.

2. Die genetische Darstellung (Genesis = Entstehung). Der Topograph erkennt an der geometrischen Form auch die Wirkung von Kräften der Tektonik, Stratigraphie und Glaziologie und sieht sich nun vor die Aufgabe gestellt, die geometrische Form unter Innehaltung der genetischen Merkmale zu zeichnen. Das gibt unter Umständen ein ganz anderes Bild, indem die Schraffen, die man ja künstlerisch ganz beliebig frei in die vorliegende geometrische Form legen kann, nun eben nach genetischen Gesichtspunkten und nicht nach orographischen $\mathrm{zu}$ liegen kommen. Dieser Prozeß hängt stark vom Sehvermögen des Topographen ab. Das Sehvermögen andererseits ist eine Funktion der Erkenntnis, die der Topograph über die Entstehung des darzustellenden Objekts besitzt. Das Matterhorn bleibt also ein Vierkanter, aber in den Flächen erkennt man an der Strichlage der Schraffen die Wirkungen der Tektonik (Falten), Stratigraphie (Schichtung) und der Glaziologie (Schliffe).

Die Unterscheidung von orographischer und genetischer Darstellung ist etwas willkürlich, aber praktisch und meint, daß man bei genetischer Darstellung speziell auf die Entstehung des Körpers, resp. ihre Resultate abstellt. Man stoße sich also nicht an dieser Willkür. Willkürlich ist sie insofern, als die fluviatile Erosion, welche hauptsächlich die orographische Form bestimmt, streng genommen ja auch eine genetische Komponente ist. Mit der Erosion rechnet aber jeder, selbst der primitivste Topograph, gleichviel ob bewußt oder unbewußt von Anbeginn an. Mit der Stratigraphie, Tektonik und Glaziologie aber ist es anders; die Darstellung der Wirkungen dieser Momente setzt erst um 1870 ein. Jeder 'Topograph, auch wenn er erst 1958 zum Zeichnen kommt, macht diese ganze Entwicklung, nur in kürzerer Zeit, an sich selber durch. Mag sein, daß er im Stadium vor 1870 stehen bleibt; dann hat der Most eben noch nicht gegoren. Die Unterscheidung von orographischer und genetischer Darstellung hat sich in unserer Praxis sehr gut bewährt. Ich habe mehrfach beobachten können, wie ein genetisch sehender Topograph anläßlich von Revisionen ein Pauspapier auf die Zeichnung eines orographisch arbeitenden Topographen legte, damit ins Feld ging, und das Blatt unter Innehaltung der geometrischen Form auf Genesis umzeichnete. Und ich habe oft genug zugeschaut, wie man Gräte und Kegel zeichnet, wo Platten lagen, daß man Bänder zeichnete, wo kompakte, glatte Granitwände trotzten ${ }^{4}$. Ich wiederhole:

4 In meiner Arbeit: Historische Entwicklung auf Plänen und topographischen Karten (Schw. Z. f. Verm. und Kulturtechnik, 1930) sind auf Tafel 2 in den Abb. 14 und 15 zwei frappante Beispiele aufgeführt. In Abb. 14 zeichnet L'HARDY im Wallis eine mächtige Felswand mit Bändersignatur, während der Genetiker IMFELD dieselbe Wand mit «Dachziegelbedeckung» darstellt. Ist wohl auch zweierlei für den Touristen, nicht bloß für den Topographen! Und in Abb. 15 zeichnet der Orograph Wolfsberger im Berner Oberland harte, markante Felsbänder, während der Genetiker BEcker am selben Ort glazial (und dies lediglich durch ganz andere Lage der Schraffen) geschliffene Wände und Böden zeichnet. L'HARDY u. WolfENSBERGER waren DUfour-Topographen, während IMFELD und BECKER die genetischen Revolutionäre der Siegfriedkarte waren. 


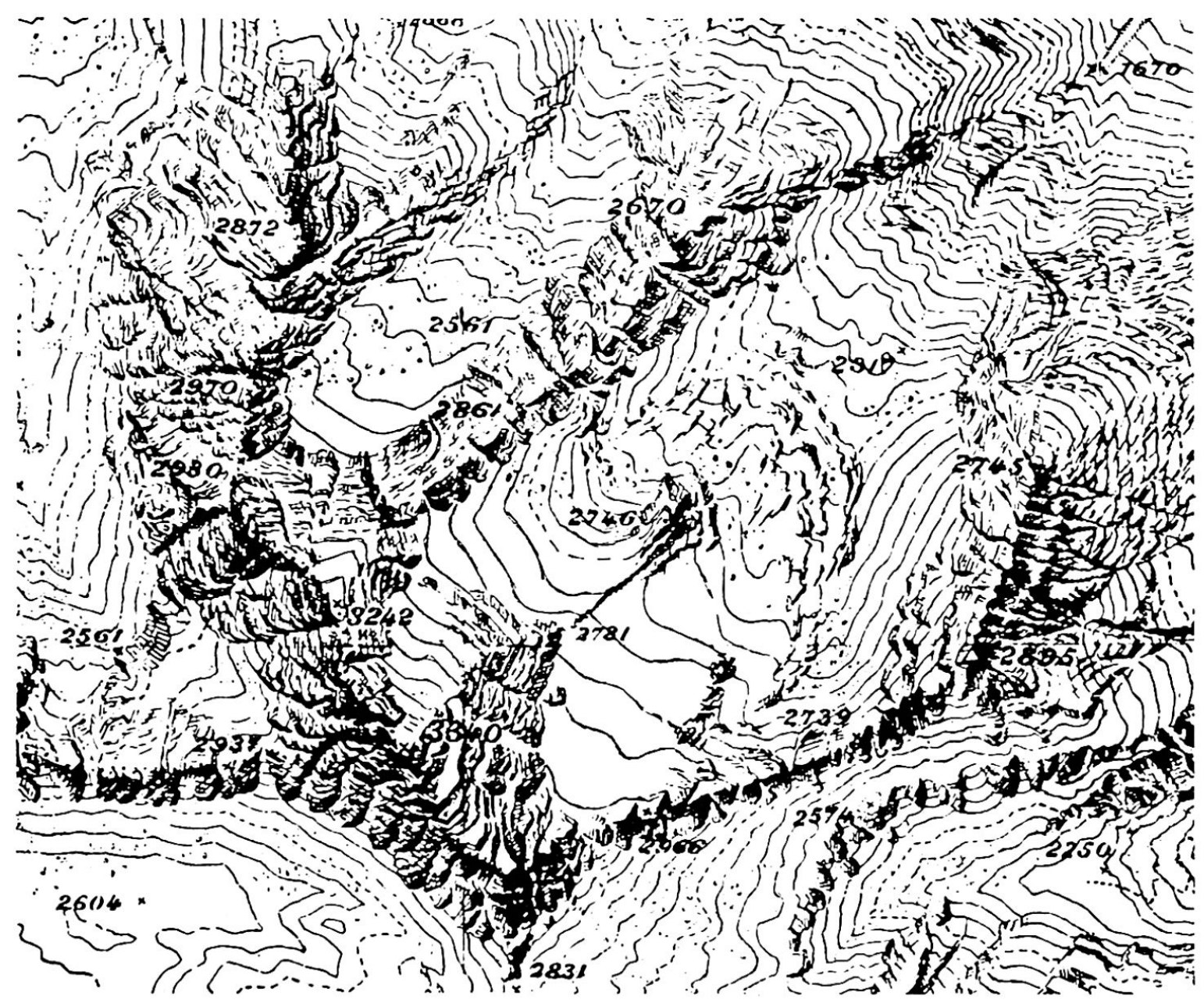

Abb. 6 Ausschnitt aus der Originalzeichnung zu Blatt Savognin SA 426. Aufnahme und Zeichnung von Held I885-86. Felsschraffen-Zeichnung. HeLd ist der spätere Direktor der Eidg. Landestopographie. 1:1

Die orographische Darstellung gibt also den Zustand ohne Rücksicht auf das Geschehen beim Entstehen der Form, klammert sich bloß an die geometrischen Tatsachen. Die genetische Darstellung stellt auf den Vorgang der Entstehung der Form ab und zeichnet die Wirkungen davon. Mag sein, daß die geometrische orographische Darstellung bereits auch die Genesis wiederspiegelt, ohne daß man es extra betont. Es ist dies aber lange nicht immer der Fall; das hängt ganz von der Schraffenlage ab. Anderseits muß eine genetische Darstellung immer zugleich der geometrischen Form gerecht bleiben; das ist mit ein Grund, warum sie als bessere Methode sich durchsetzen muß.

Orographische Beispiele haben für mich bloß noch historischen Wert. Es sollte nach meiner Ansicht nicht mehr vorkommen, daß ein Topograph orographisch zeichnet, wo genetische Möglichkeiten bestehen. Orographische Beispiele gehören also auch in eine historische Abhandlung. Sie wirken meistens auch hölzern, nicht felsig.

Zum Thema der Schraffendarstellung sei weiterhin folgendes ausgeführt:

Wenn H. Dufour in seinen «Reconnaissances militaires» irgendwo sagt: «Ce qu'il-y-a de plus difficile dans le dessin militaire, c'est sans contredit la représentation des rochers. Il n'est pas possible de poser d'autres règles à ce sujet, que de s'astreindre à imiter ce qu'on voit» (Dufour, G. H.: Instruction sur le dessin des reconnaissances militaires à l'usage des officiers de l'école fédérale. Genève 1828),

so ist das richtig, genügt aber nicht; denn jeder sieht etwas anders als sein Nachbar, und dies, wie schon gesagt, entsprechend dem Grade der Erkenntnis, die er von der Sache hat. 

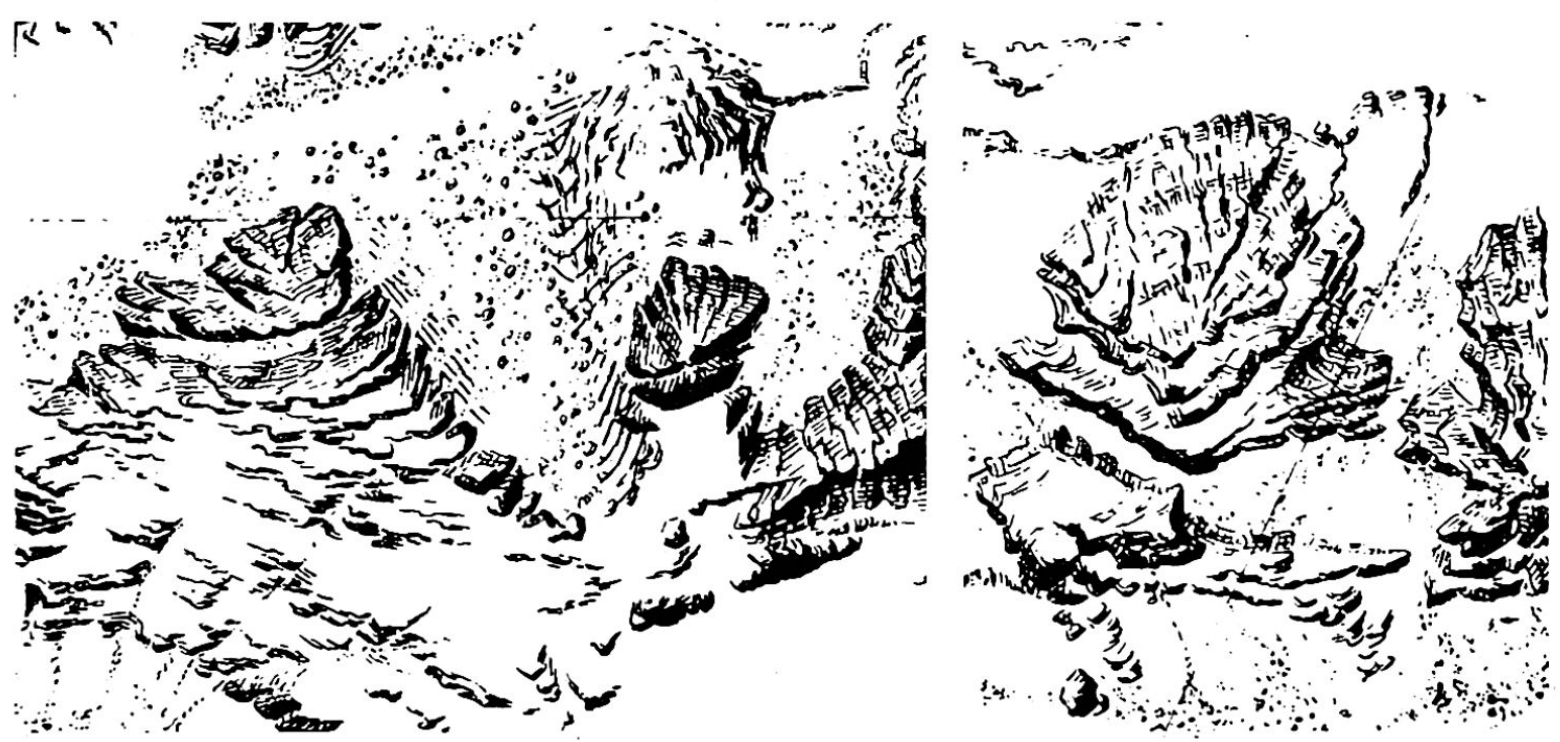

Abb. 7 Ausschnitt aus der Originalzeichnung zu einer Revision der Felszeichnung zu Blatt Etivaz SA 469, Revisionspause. Aufnahme und Zeichnung von Held. Felsschraffen-Zeichnung. Verkleinert

Die zitierte Anleitung zum Felszeichnen von Dufour deckt sich mit der Anleitung zum Freihandzeichnen, die H. Pestalozzi in seiner Schrift: «Wie Gertrud ihre Kinder lehrt», zeitlich etwas früher publiziert hat. Er schreibt:

«Zeichnungskunst ist eine Fertigkeit, sich den Umriß und die innert demselben enthaltenen Merkmale eines jeden Gegenstandes, durch Anschauung des Gegenstandes selber, mittelst ähnlicher Linien vorstellen und sie getreu nachahmen zu können.»

Im Bestreben nun, den Topographen sehen zu lernen, hat man versucht, typische Felsbilder als Musterzeichnungen vorzulegen. Selbst Dufour, der, wie oben gezeigt, sich auf bloßes Sehen verlegte, hat zwei solcher Typen in seinen «Reconnaissances》 eigenhändig gezeichnet: 1. Rochers entrecoupés de paturages; 2. Rochers grandes masses et en couches plus paralleles. Als ehemaligem Schüler der Ecole Polytechnique in Paris waren ihm die «notions géologiques» natürlich nicht unbekannt. Die Idee hat sich denn auch seit DUFOUR mit der fortschreitenden Erkenntnis auf dem Gebiete der Geomorphologie rasch weiter entwickelt; dies besonders in Richtung Genesis. Aber auch hier möchten wir gerne auf Pestalozzi zurückgreifen.

Er schreibt in seiner bereits zitierten Schrift: «Durch das Zusammenstellen von Gegenständen, deren Wesen das nähmliche ist, wird deine Einsicht über die innere Wahrheit derselben wesentlich und allgemein erweitert, geschärft und gesichert.»

Ist es nicht wiederum so, wie wenn die Ansicht Dufours über diese Sache direkt aus diesem Satze von Pestalozzi hervorgehen würde? Dies besonders, wenn man noch die nachfolgenden weiteren Präzisierungen über das Thema, die in der eidgenössisch topographischen Instruktion, von DUFOUR stammend, abgedruckt sind:

«In diesem Sinne wird der aufnehmende Ingenieur vor allem die Terrainbildung so weit studieren, $\mathrm{da} B$ er sich über die Art und Weise der Modellierung unserer heutigen Bodenoberfläche klar wird. Er wird dabei eine Reihe immer wiedcrkehrender typischer Formen finden, die unter gleichen Gesetzen gebildet, gleichartige Gestaltung zeigen und zu deren charakteristischer Darstellung jeweilen analoge Punktbestimmungen notwendig sind. Aus der Beachtung der wechselseitigen Beziehungen zwischen geologischer Bildung, örtlicher Lage, Vegetationscharakter, Besiedlung, Wegnetz, überhaupt der gesamten Bodenbedeckung ergibt sich für den Aufnehmenden eine geistige Auffassung, welche ihn befähigt, die technischen Operationen mit Verständnis auszuführen und, bei Anbringung aller dem Maßstab zustehenden Details, e:n klares Kartenbild zu bieten.»

Einst wurde von mir das Karrenfeld in diesem Sinne nach Pestalozzi-Dufour behandelt. Heute erlebe ich nun bei der Erstellung eines Kar-Atlasses mit Pinsel und Stift alltäglich neue Überraschungen und Belehrungen im gleichen Sinne. Die Abbildungen 6, 10, 11 und 12 zeigen solche Kare als Formtypen. Auch in den meisten 


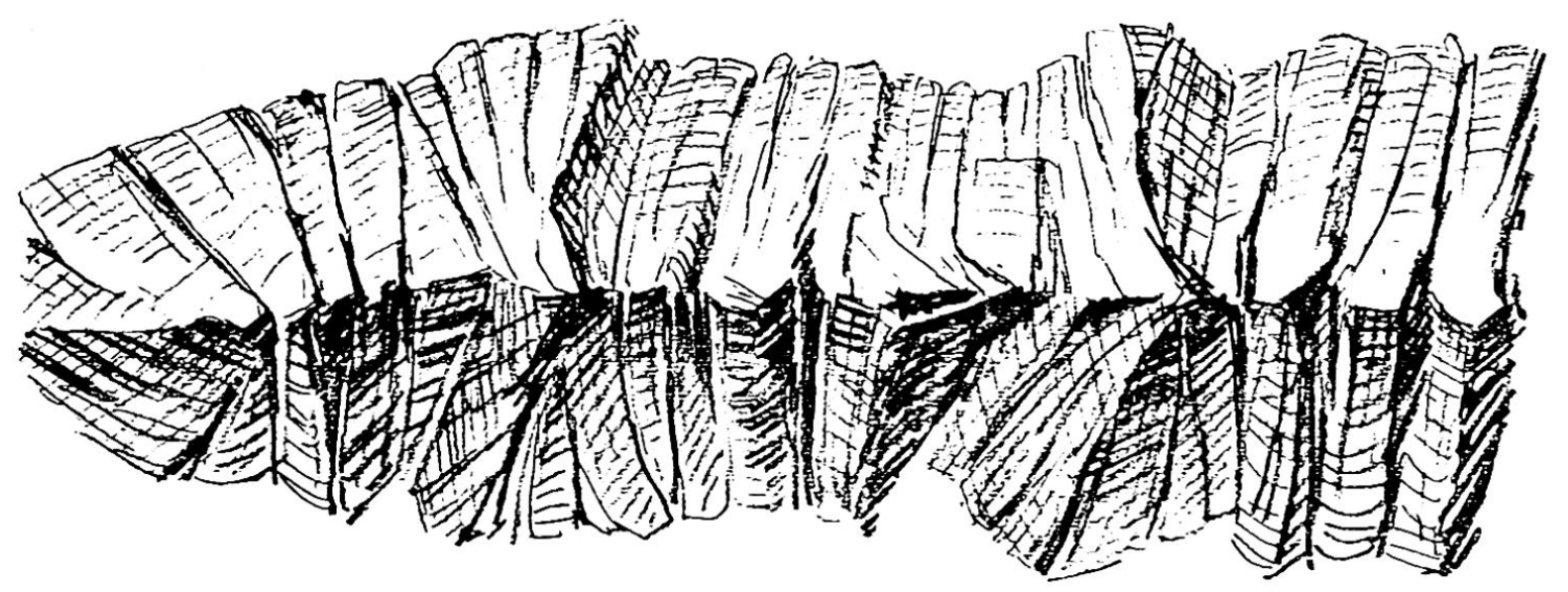

Abb. 8 Ausschnitt aus der eidg. «Instruktion 88 ». Nachzeichnung durch den Verfasser. FelsschraffenZeichnung. Kristalliner Grat in Zentralmassiv. Verkleinert

übrigen Abbildungen sind deutlich Kare zu sehen. Das Kar ist in den Alpen ein sehr häufiger Formtyp und gar nicht selten in reiner Form anzutreffen.

Praktisch muß man sich natürlich klar sein, daß solch topographische Typisierungsbestrebungen lediglich Forschungs- und Instruktionswert besitzen. Nachdem der Anfänger sich im Felszeichnen mit der Wirkung der Horizontalen, Vertikalen und der Schiefe im Schraffenbild auseinandergesetzt hat, soll er ruhig beginnen, solche Felstypenbilder zu kopieren, um seine Formenelemente anzuwenden. Erst später gestattete man ihm, sich am Objekt im Gelände zu üben. Bald wird der Moment kommen, wo er nicht mehr an seine Typen denkt, sondern als Sehender aus der Anschauung heraus gestaltet. Dann hat er den Grad erreicht, wo die Kunst anfängt, die Wissenschaft in den Hintergrund tritt. Das ist dann vollendete Topographie, so wie sie IMFELD und BECKeR ausgeübt haben. Nie aber wird man Formtypzeichnungen als Signaturen verwenden. Die Felsdarstellung hat in der Topographie die Signaturenperiode längst überwunden. Jetzt wird streng individuell dargestellt. Aber eine Signaturenperiode, wie sie in den andern Kartenelementen heute noch an der Tagesordnung ist, gab es in der Felsdarstellung auch einmal. Sie liegt aber vor 1800 . Abschließend sei nochmals betont, daß die Auffassung der physischen Form auf die Strichlage einen entscheidenden Einfluß hat.

b) Beleuchtung. Die Schraffe ist auch der Träger der Beleuchtung. Durch Variation der Strichstärke und des Strichabstandes wird meistens nach schiefer Beleuchtung gezeichnet. Für steilen Fels kommt überhaupt nur sie in Frage. Bei flacheren Partien könnte man über senkrechte Beleuchtung diskutieren. Die Diskussion würde aber kaum ein positives Resultat ergeben.

c) Luftperspektive. Auch für sie ist die Schraffe der Träger. Wie die Beleuchtung wird sie durch Variation der Strichdicke und des Strichabstandes gemeistert. Sie äußert sich so, daß gegen den Talboden hin die Gegensätze zwischen Licht und Schatten abnehmen und alles mehr im selben Grauton versinkt (Abb. 14).

Diese alte Felszeichnungsmethode mit Schraffen ist keineswegs einfach zu handhaben. Die Schwierigkeit besteht darin, daß die Fläche, bis auf die hellsten Partien, gänzlich mit Schraffen belegt sein muß und da $\beta$ in einem $G u ß$, sozusagen bei jedem Strich, gleichzeitig die drei Komponenten Auffassung, Beleuchtung und Luftperspektive gemeistert werden müssen, damit man zur physischen Form gelangt. Man muß also die Feder fortwährend nach der Resultanten dieser drei Komponenten führen.

\section{DIE NEUE PHOTOGRAMMETRISCHE METHODE}

Diese neue Methode kann nur richtig gehandhabt werden, wenn das soeben über die herkömmliche Schraffenmethode Gesagte verstanden wurde. Die Komponenten der 


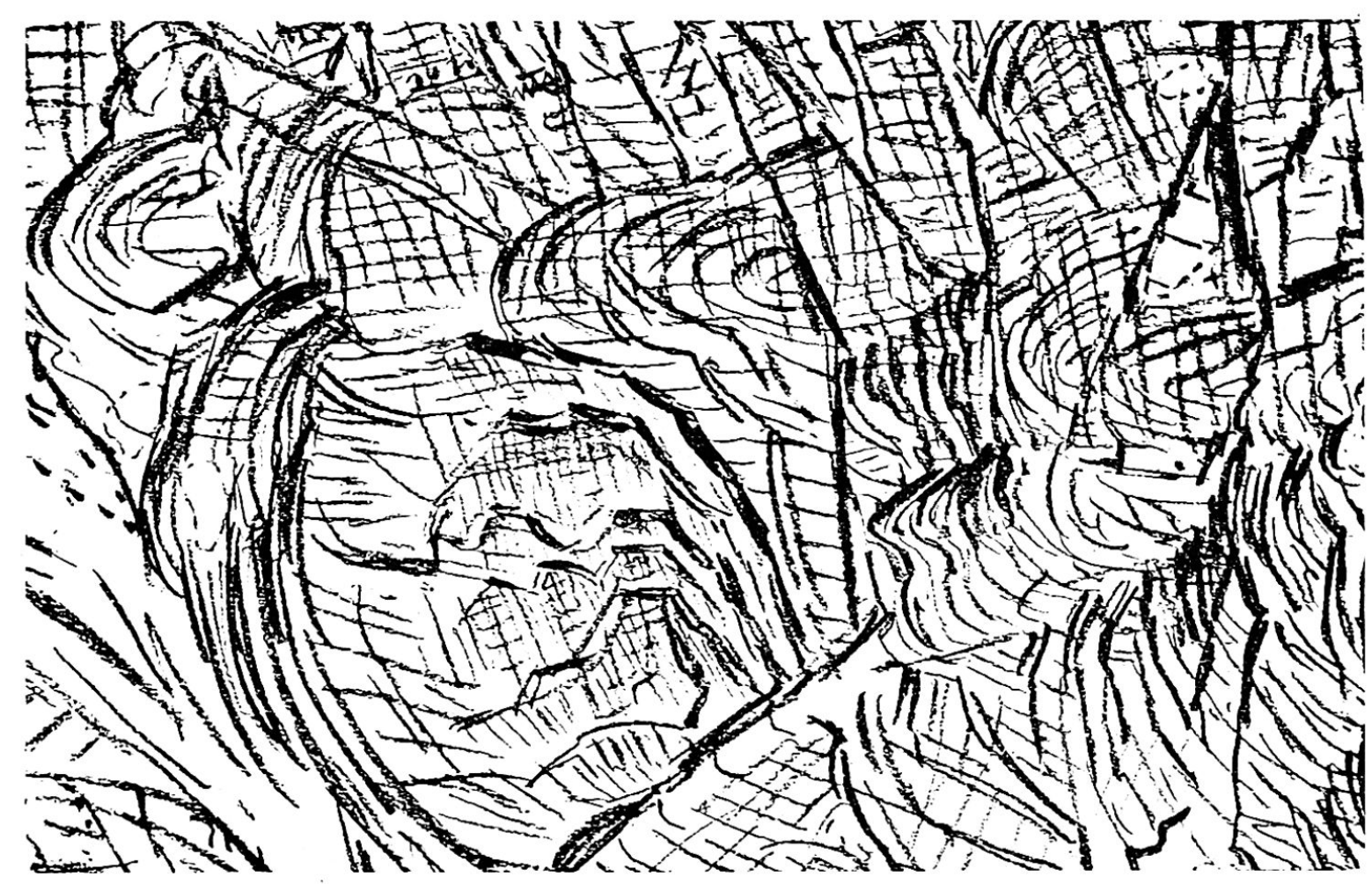

Abb. 9 Ausschnitt aus der kartographischen Bearbeitung des Übersichtsplanes Gcharretealp, Kt. Schwyz. Zeichnung vom Verfasser. Felsschraffen Zeichnung. Schwachgeneigte Kalkplatten als Schichtköpfe. Verkleinert

neuen Methode sind absolut die gleichen; alles früher Gesagte gilt sinngemäß auch für sie, und die Darstellungsmittel sind andere.

Die Felskurve als Höhenkurve (Abb. 8). Am Auswertegerät können beliebig viele und beliebig genaue Felskurven herausgegeben werden. In photogrammetrischen Auswertungen ziehe man sie so dicht, wie sie kommen und lasse sie ruhig da, wo sich die Aequidistanz als zu eng erweist, ineinanderlaufen. Diese enge Kurvenscharung ist dem Topographen für die Zeichnung der Felslinien sehr dienlich. Je totaler die Fläche von den Kurven integriert wird, umso bessere Grundlagen enthält sie für die Zeichnung.

Aber nicht nur der Topograph, sondern auch der Photogrammeter-Geodät sieht diese ineinanderlaufenden Kurven gerne. Erstens hat er bei der Auswertung derselben ein kontinuierliches, fließendes Arbeiten und dazu kommt, daß solche Kurvenagglomerationen einen außerordentlich bestrickenden graphischen Vorteil haben. Die Färbung des Papieres durch die Kurven geschieht nämlich nach der Regel: je steiler das Gelände, umso farbensatter, resp. dunkler die Papierfärbung. Die weißen Intervalle zwischen den Kurven kommen einem Aufhellen des Farbtones der Kurven mit Weiß, gleich. Dieses Aufhellen des Farbtones nach der Steilheit des Terrains geschieht zu dem in geometrisch exaktester und erst noch automatischer Weise. Kartographisch gesprochen ist das nichts anderes als eine exakt vorbereitete senkrechte Beleuchtung. Der Kartograph hat damit gewonnenes Spiel, er muß diese senkrechte Beleuchtung bloß noch zwischen den Kurven physisch ergänzen, und er hat eine ungeahnt genaue senkrechte Beleuchtung seiner Terrainoberfläche. Zudem ergibt diese Methode, besonders für Terraingebrauch, aber auch für alle Arten von Terrainanalysen im Bureau eine außergewöhnlich gute Darstellung. Bildhaft auf den ersten Blick ist sie allerdings nur bei bestimmten Terrainformationen, nie aber versagt sie. Sie ist die zuverlässigste und wissenschaftlichste Methode der Terraindarstellung überhaupt. Tatsächlich sagen auch alle Leute mit Felderfahrung, daß man mit dieser Beleuchtung weit besser fährt im Terrain als mit der schiefen Beleuchtung. Das kommt da- 


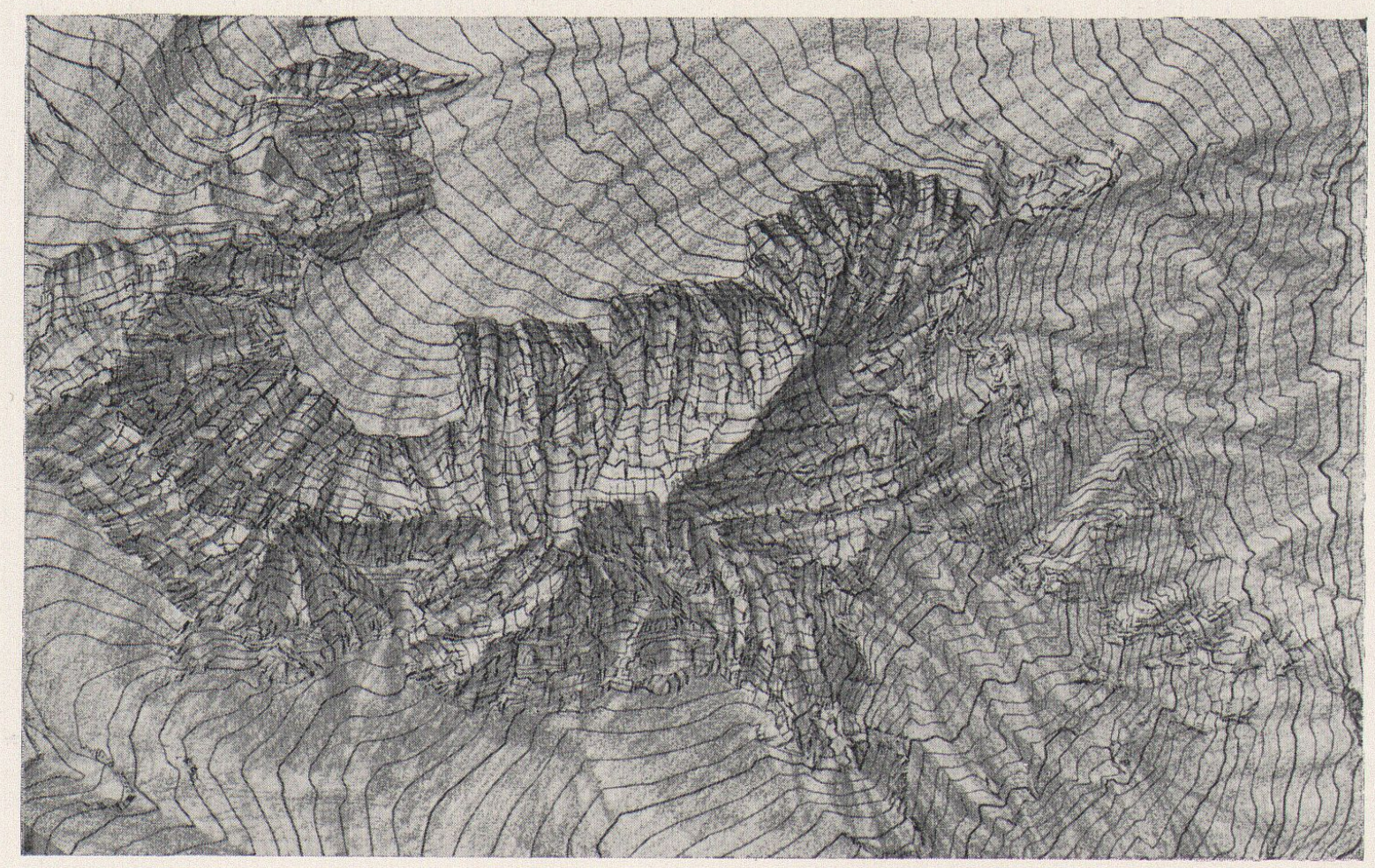

Abb. 16 Ausschnitt aus dem Übersichtsplan Val Ferret. Treutse Bo. Äquidistanz $20 \mathrm{~m}$. Felskurven, Felslinien und Reliefton. Zeichnung und Bemalung vom Verfasser. Zu stark verkleinert; muß bereits mit der Lupe gelesen werden, weil Originalzeichnung sehr fein gehalten ist



Abb. 17 Ausschnitt aus dem Übersichtsplan Mürtschenstock. Äquidistanz 20 m. Felskurven, Felslinien und Reliefton. Zeichnung und Bemalung vom Verfasser. Verkleinert 


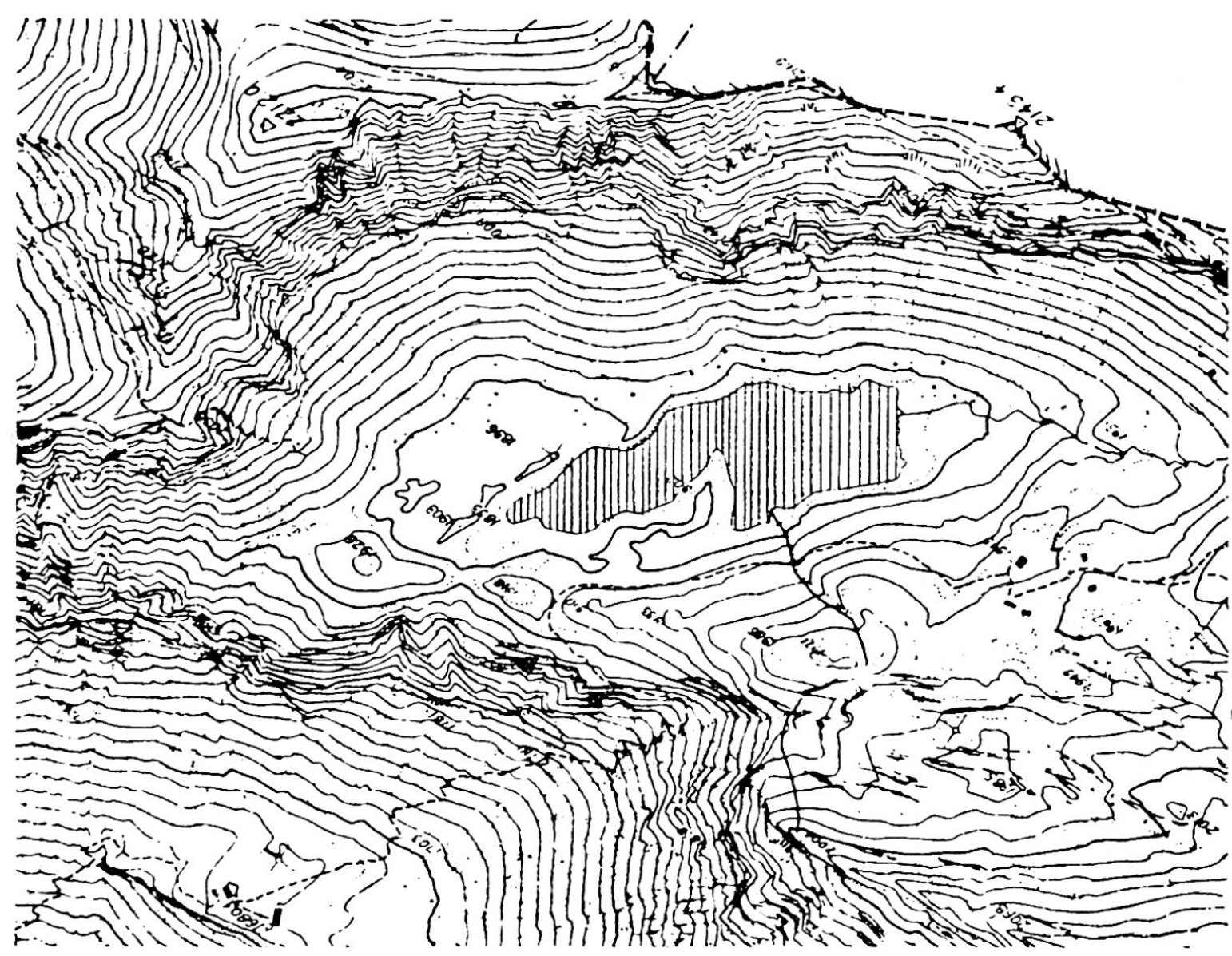

Abb. 10 Ausschnitt aus dem Übersichtsplan Flüeli. Eiseekar auf der Nordseite der Brienzer-Rothornkette. Bloße Felskurven. Der Ausschnitt ist nach Südosten, anstatt nach Norden orientiert. Damit wird der Blick des Beschauers nicht über die Rücklehne, sondern durch die Öffnung in das

Kar gelenkt. Es zeigt sich, daß man dadurch die Kesselform des Kares viel leichter erfaßt

von her, weil man im Terrain die natürliche Oberfläche ja bereits vor sich hat, man braucht gar keine Interpretation nach schiefer Beleuchtung, die Analyse nach der Neigung ist viel wichtiger.

Praktisch arbeitet der Photogrammeter-Geodät also ganz einfach nach dem Rezept, das Friedrich DER Grosse seinem Militäringenieur Müller gab: «Wo ich nicht durchkomme, mache er einen Klecks!» Tatsächlich klecksen des Photogrammeters Kurven überall dort, wo die Klettereien höherer Grade anfangen. Daraus folgt, daß diese senkrechte Beleuchtung die beste Darstellungsart für Kommunikationen ist. Der Zivil-Ingenieur, der solche projektieren muß (Wege, Straßen, Bahnen), wird mit Vorteil senkrechte Beleuchtung als Grundlage verwenden; ebenso der Wanderer, der nicht speziell klettern will. Wir haben in Abb. 9 die wenigen existierenden Wege jener Karlandschaft mit dickem Strich eingezeichnet. Wie prächtig legt sich das Trasse des Überganges von der Leventina in die Val Verzasca am Laghetto ins Gelände. Müßte man den Weg erst trassieren, man käme bei senkrechter Beleuchtung sehr rasch auf dieselbe Lösung, man muß nur die farbensatten, dunklen Stellen meiden.

Klar ist zum vorneherein, daß man solche senkrechte Beleuchtungen in jeder beliebigen Farbe drucken kann. Zur Zeit Friedrichs des Grossen und lange nachher, wurde die senkrechte Beleuchtung nur schwarz gedruckt. Das gab für viele Karten, besonders Gebirgskarten, eine katastrophale Schwärzung des Papieres. Gegen Ende des letzen Jahrhunderts wurde dann oft in rotbraun gedruckt, was sich sehr bewährt hat. Am Übersichtsplan 1:10 000 wird Oliv, das sich ebenfalls sehr gut bewährt hat, vorgezogen. Gibt man den Kurven dieselbe Farbe wie dem ergänzenden Schummer- 


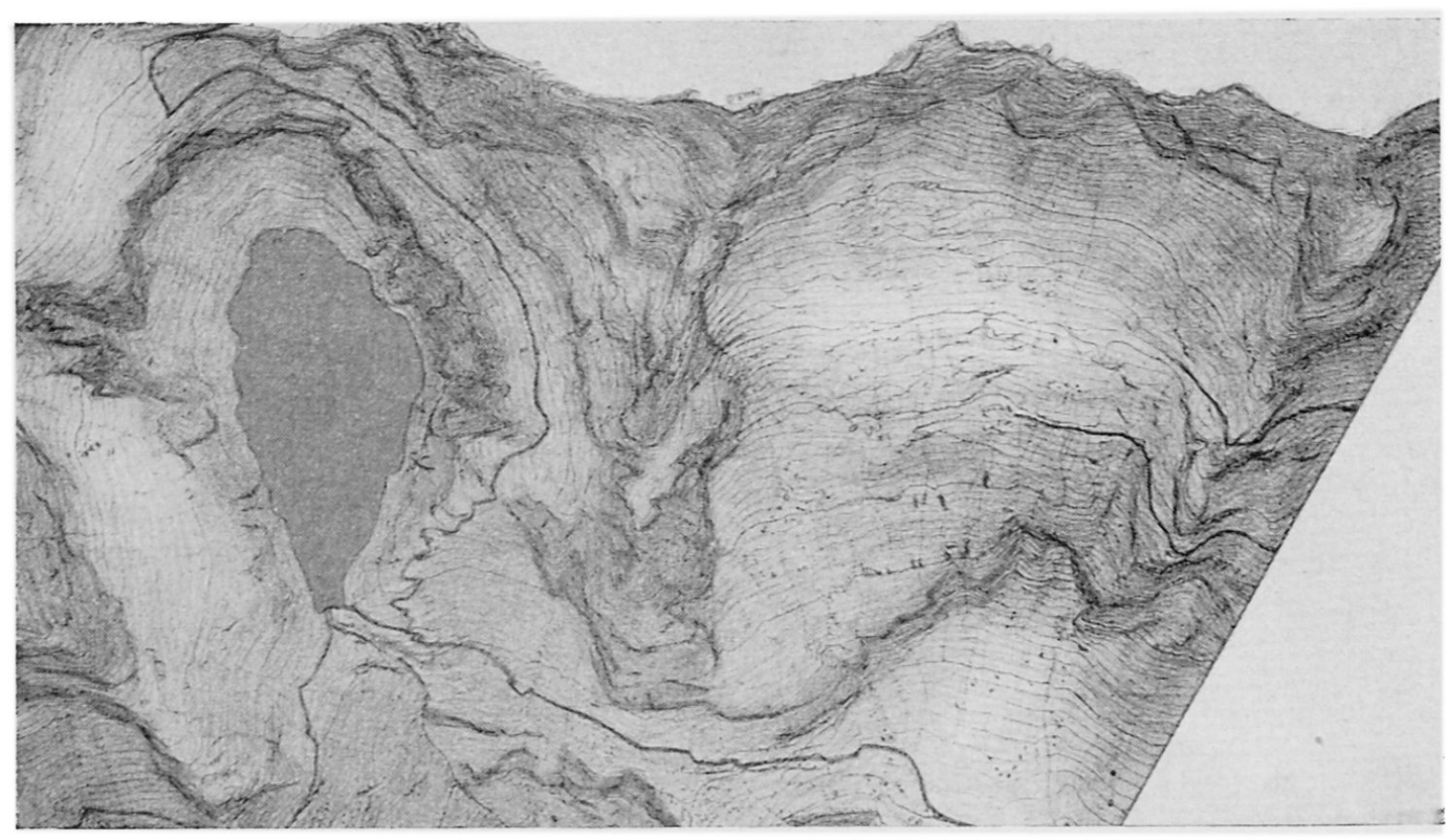

Abb. 11 Querschnitt aus dem Übersichtsplan Chironico. Laghetto-Trog und Kar von Campala. Bloße Felskurven mit senkrechter Beleuchtung. Bemalung vom Verfasser. Stark verkleinert

ton, z. B. rot, wie ich es im Original zu Abb. 11 gemacht habe, so erhält man rasch ein kontinuierlich laufendes physisches Terrainbild in senkrechter Beleuchtung.

In meinem Kar-Atlas male ich aber ruhig in allen Spektral- und Naturfarben; ja noch mehr, ich verwende in der Neigungsskala mehrere Farben nebeneinander. Dies ist bei Karlandschaften besonders ausdrucksvoll. Man muß nur die Farbwechsel in die hier allgemein sich vorfindende scharfen Gefällsbrüche legen, was sich beinahe von selbst ergibt.

Es hat sich beim Malen solch buntfarbiger senkrechter Beleuchtungen bei mir noch folgendes gezeigt: Sobald man in Gratlandschaften, wie in den Alpen, malt, leistet auch die buntfarbige senkrechte Beleuchtung nicht alles. Ich pflege dann lasierend eine schiefe Beleuchtung darüber zu malen und habe für das Endprodukt dann eine Doppelbeleuchtung. Ausgezeichnet bewährt hat sich dabei, die senkrechte Beleuchtung in Orange zu malen und die schiefe Beleuchtung in Violett oder Sepia darüber zu legen. Die schiefe Beleuchtung paßt bei solchen Doppelbeleuchtungen dann sehr gut $\mathrm{zu}$ der schiefen Beleuchtung, die ich bereits in der Felszeichnung drin habe. Und auch sonst ergänzen sich die zwei Beleuchtungen, senkrecht und schief, in der Doppelbeleuchtung erstaunlich gut. Man hat in der Doppelbeleuchtung die Vorteile beider Beleuchtungen, der senkrechten und der schiefen, und ..... eine hebt die Nachteile der andern auf, ohne daß sie sich auch nur im geringsten stören. Wer die Diskussion um die Nach- und Vorteile der senkrechten und der schiefen Beleuchtung kennt, weiß sehr wohl, was das bedeutet. Es erübrigt sich, das im Detail hier auszumalen. Wohl aber will ich noch ein paar Worte über den hier so häufig verwendeten Ausdruck «senkrechte Beleuchtung» anfügen. Es ist nämlich höchst ungenau, bei der Schattierung durch Horizontalkurven von senkrechter Beleuchtung zu reden. Wenn dann noch in derselben Skala mehrfarbig gemalt wird, wie ich es oft mache, so kann man sich die Sache als Effekt eines Beleuchtungsvorganges schlechthin ganz und gar nicht mehr vorstellen. Das aber gilt für alle «senkrechten Beleuchtungen», denn die in der Praxis üblichen beruhen alle mehr auf Konventionen, denn als auf tatsächlichen Beleuchtungseffekten. Ich verwende daher zu genauerer Bezeichnung dieser Darstellungsverfahren die Deklaration «Terraindarstellung in 
Gradation der Neigung». Es ist aber keineswegs nötig, sich immer so genau auszudrücken. Die Bezeichnung «senkrechte Beleuchtung》 ist seit 150 Jahren in der Kartopographie derart allgemein geläufig, daß sie ruhig als Sammelbegriff für alle solchen Böschungsdarstellungen weiter verwendet werden darf.

Damit komme ich zum Schlusse meiner Diskussion um die Kurvenagglomerate des Photogrammeters, welche mich auf die senkrechte Beleuchtung gebracht hat. Eine reine Kurvenzeichnung kann in Felsgebieten weder in Karten noch in Plänen je als eine fertige Felszeichnung angesprochen werden; es gehören mindestens noch Felslinien dazu. Und da es in der Kartographie nie und nimmer gelingen wird, Felsgebiete in senkrechter Beleuchtung darzustellen, muß man bei allen Karten mit senkrechter Beleuchtung das Felsgebiet sofort ausschließen, um schiefe Beleuchtung zu verwenden. Die Praxis zeigt, da $\beta$ diese Konzession ganz gut geht, zum Erstaunen gut. Felsen sind eben so detailliert, daß man sich gar nicht stört daran, daß eine andere Beleuchtung herrscht als im übrigen Terrain. Das Auge wird vom ersten Moment an derart durch die anschauliche, für Fels so sehr passende schiefe Beleuchtung gefesselt und direkt beschäftigt, daß für eine kritische Überlegung wegen der Beleuchtung gar keine Zeit bleibt. Dazu müssen aber die Kurvenagglomerationen aus dem definitiven Kartenbild unbedingt verschwinden. Das Kurvenbild sollte also wohl durchgehend gezogen werden, aber für die Reproduktion ist es aufzulockern. Wann das im photogrammetrischen Arbeitsprozeß geschieht, werden wir später sehen. Das Wie aber lautet so: Man zeichne zuerst die Leitkurven; was enger als $0,2 \mathrm{~m} / \mathrm{m} \mathrm{zu}$ sammenkommt, wird weggelassen. Eine Beleuchtung der Kurven durch Verdickung derselben auf der Schattenseite hat sich bei unseren diesbezüglichen Versuchen nicht bewährt. In Spezialfällen mag es ein nützlicher Kniff sein, um zu einer gewissen Reliefwirkung zu kommen.

Felslinien. Gerippezeichnung empfahl J. M. ZIEGLER schon 1862. Er charakterisiert bei nackten Felspartien mittelst Umrissen und leichten Strichen Schichtung und Lagerung des Gesteins. Diese Felsdarstellung denkt er sich für den Handgebrauch des Technikers, Geologen und Naturforschers und nennt die betreflende Kartenausgabe «Detailkarte» 5 .

Später, im Jahre 1910, hat Prof. Becker- bei der Auszeichnung seiner Meßtisch-photogrammetrischen Aufnahme des Mürtschenstocks, solche Gerippelinien verwendet. Ich habe einen Ausschnitt davon auf Tafel III als Abb. 18 in meiner Arbeit unter Anmerkung 4 im Jahre 1930 veröffentlicht. Hier treten die Gerippelinien zum ersten $\mathrm{Mal}$ in Verbindung von Felskurven auf.

Die Gerippelinien, ich nenne sie lieber Felslinien, sind die Kanten oder Verschneidungslinien der Flächen und die auffallenden morphologischen Linien, die der Topograph entweder explizit oder implizit bereits bei seinen Schraffenlinien verwendet. Explizit insofern, als er die Ränder der topographischen Felsflächen und der führenden Linien darin, z. B. in einer Falte, wirklich auch im Schraffenbild als Linien zeichnet. Implizit insofern, als er diese Linien und Ränder als Lichtkanten oder Schraffenunterschiede indirekt erzeugt. Bei der neuen Methkode zeichnen wir sie meistens explizit; selten figurieren sie indirekt. Indirekt sind sie dann, wenn wir sie durch Scharung der Kurven in einer immer wiederkehrenden Knickung in denselben angedeutet sein lassen oder einen Schattenton an eine solche Linie heranführen, ohne diese Linie selbst zu zeichnen. Daß man bei der Zeichnung dieser Felslinien rein orographisch oder aber mehr genetisch vorgehen kann, ist klar.

Gelingt es, mit solchen Linien ein vollständiges Felsgerippe zu zeichnen, das nachher ohne Kurven und ohne andere Hilfsmittel, so wie ein Drahtgestell, selbst stehen kann, ohne umzufallen, dann ist es umso besser. Denn das Hauptelement in der fer-

5 J. M. Ziegler: Über topographische Karten in großem Maßstab und die Karte des Kantons Glarus insbesondere, mit 4 reduzierten Karten 1:25 000 , Winterthur, J. Wurster \& Co. 1862. ZIEGLER finanzierte 1842 in Winterthur die Gründung des geographisch-kartographischen Institutes von WURSTER \& Co. und war dessen wissenschaftlicher Leiter. Das Institut erreichte bald Weltruf, existiert aber heute nicht mehr. 
tigen Felsdarstellung sollte diese Felslinienzeichnung sein. Die Kurven können dieses System der Felslinien lediglich sinnvoll füllen und müssen sich diesem Zwecke unterordnen, ähnlich wie bei der Schraffendarstellung die Felsschraffe die Fazetten füllte. Hat man Felsen, die innerhalb der Flächen keine Felslinien anbieten, so lasse man ruhig die Kurven die Flächen integrieren. Das ist nicht ein Nachteil, sondern ein großer Vorteil dieser Methode. Wo hingegen die felsige Gestaltung stark akzentuiert ist, erfasse man mit den Felslinien so viel Details als der Maßstab erlaubt, glatte Wände also lediglich durch Umrandung, gebänderte Abstürze mit mehr Felslinien. Schwieriger wird es, wenn die orographischen und genetischen Felslinien horizontal laufen wie die Kurven, was recht häufig vorkommt. Haben die Linien zwischen den Kurven Platz, dann ist es gut. In einfarbiger Wiedergabe ist es aber auch so noch schwierig, die Felslinien von den Kurven zu unterscheiden. Das kommt besonders leicht bei horizontalen Bändern vor, wo man gerne den unteren und oberen Rand als laufende Linien angeben möchte. Man kann sich in solchen Fällen aber immer noch helfen. Man teilt am besten die Bänder in einzelne Fazetten auf, die man mit vertikalen Linien abgrenzen kann; zudem kann man die unteren und oberen Abgrenzungen mit diesen Vertikalen binden und dadurch von den Kurven unterscheiden: Ebenso wird man horizontale obere und untere Ränder von Wänden im Stich nicht glatt durchziehen, sondern auflösen und irgendwie mit der Innenfläche in Beziehung setzen, um sie von den Kurven zu unterscheiden. Im übrigen ist ja glücklicherweise die Erosion des fließenden Wassers unser Hauptformbildner. Sie arbeitet in der Regel vertikal und ist daher mit horizontalen Kurven gut faßbar.

Felslinien wird man am Autographen als punktierte Linien soweit auswerten, als es der Topograph verlangt. Dies gilt auch bei der Schraffendarstellung. Es ist eine ärgerliche Arbeit, wenn man beim Felszeichnen immer wieder versuchen muß, solche fehlende Felslinien festzulegen. In steilem Gebiet geht es der vielen Kurven und Formen wegen leichter als in flachen Gebieten, wo es oft unmöglich ist, ohne zusätzliche Meßoperationen zum Ziele zu kommen. Es scheint uns aber falsch, wenn der Topograph bei der Reambulierung mit Visuren, Durchstoßpunkten oder gar Lattenpunkten diese Linien nachträglich im Feld bestimmen muß, wo man sie leicht und bequem am Auswertegerät herausgeben kann. Der Verfasser hat z. B. große Karrengebiete bearbeitet, bei welchen schüchtern einige durchgehende Spalten in den verkarrten Kalkplatten ausgewertet waren. Es bestanden aber deren zu wenige und die Auswahl war schlecht. Nach der Auswertung allein konnte man das Felsbild nicht richtig zeichnen.

Um die Plastik des Liniengerippes zu heben, wird man auf der Schattseite das Liniennetz etwas kräftiger ausführen als auf der Sonnseite.

Für die Reproduktion wird man das Felsgerippe möglichst auf die Schwarzplatte nehmen, denn scharz zeichnet am schärfsten und auffallendsten, was der Bedeutung des Liniengerippes im Kartenbild entspricht. Unsere Versuche zeigen aber, daß man vorteilhaft auch die Felskurven schwarz mitdruckt, sie allerdings so fein als möglich. Dadurch kommt man viel besser zu einem zusammenhängenden Felsbild als seinerzeit bei der Schraffenzeichnung.

Zur Herstellung solcher Felslinienzeichnungen sei folgendes bemerkt: Ich habe als Ingenieur der Landestopographie jahrzehntelang an den Auswertungen am Autographen teilgenommen. Damals war es üblich, auf Glasplatten in Negativgravur auszuwerten. Man kann so von der Auswertung im Kontaktverfahren sofort Kopien haben, ohne zu photographieren. Das ist zweifellos ein Vorteil; aber der Vorteil ist nicht so groß, wie es auf den ersten Blick scheint; denn diese Auswertungen sind als kartographisches Produkt nicht direkt verwendbar und höchstens für analytisches Arbeiten geeignet. Man muß also dem Topographen eine Kopie geben, damit er das Autographenblatt im Feld ergänzen kann. Hierauf muß es von demselben ausgezo- 


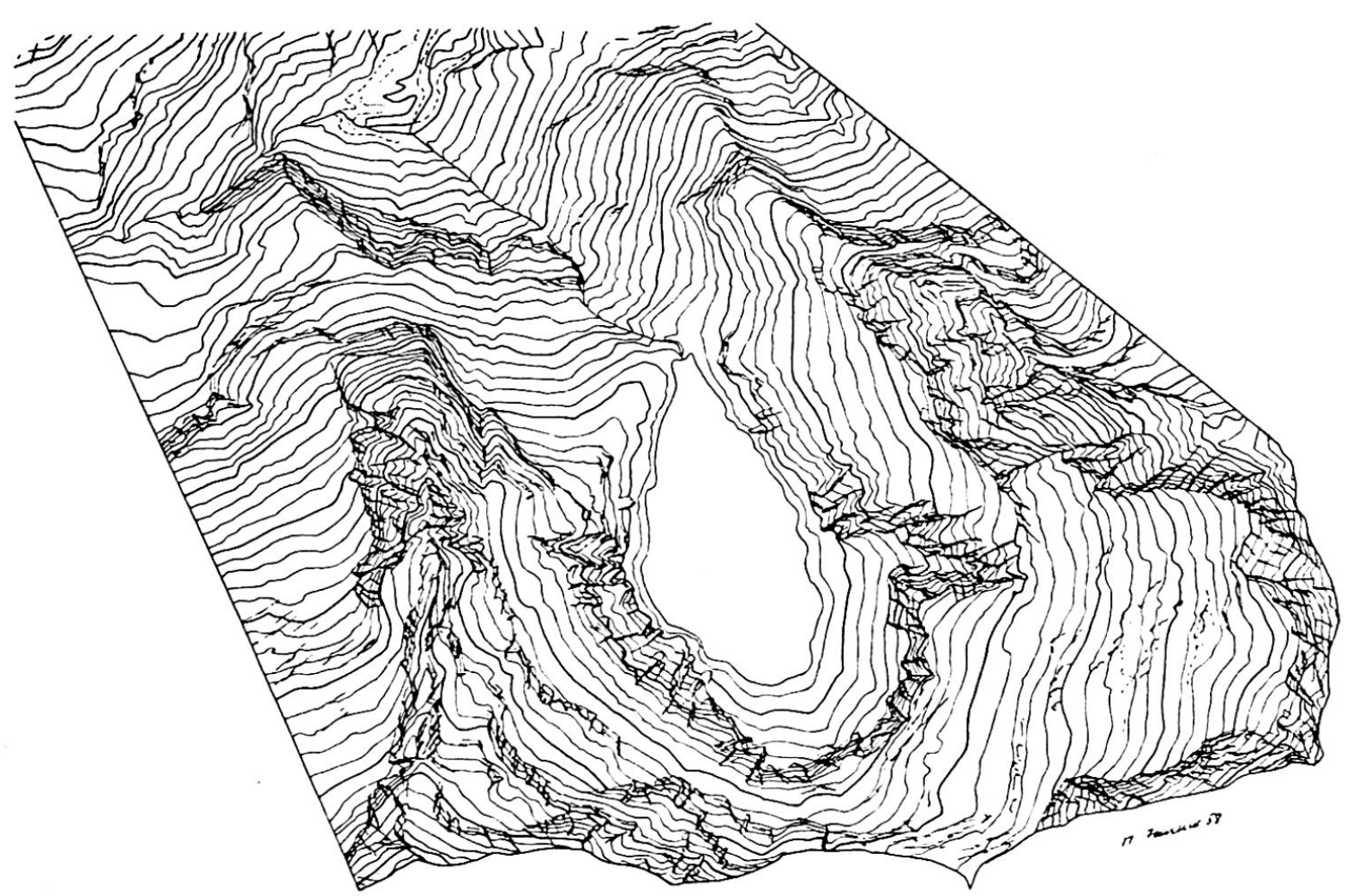

Abb. 12 Ausschnitt aus dem Übersichtsplan Chironico. Laghetto-Trog. Felskurven mit Felslinien. Zeichnung vom Verfasser. Stark verkleinert

gen werden, vor allem auch bei verlangter Mehrfarbigkeit. Will man nun weitere Kopien, so muß man doch auch photographieren.

Bei der Grundbuchvermessung ist der Prozeß ein anderer. Dort wertet man anstatt in Negativgravur auf Glas lieber mit Bleistiften auf Papier aus. Das Autographenoriginal dient dann gleich als Feldoriginal für die Ergänzung und wird sofort nach der Autographenprozedur mehrfarbig ausgezogen. Hierauf wird photographiert und auf die Gravurplatte übertragen, um es zu gravieren.

Mir scheint, daß man in beiden Fällen darnach trachten sollte, das Autographenoriginal so $\mathrm{zu}$ gestalten, daß es möglichst bald als Kartographenoriginal verwendet werden kann, dies aus zwei Gründen:

1. Die Autographenoriginale sind sehr begehrt, und man kann oft nicht zuwarten, bis sie in zusammenhängenden Blättern reproduziert sind; sie werden oft noch in warmem Zustand verwendet!

2. Die Redaktion der Blätter muß abgeschlossen sein, bevor das Blatt zum Graveur geht. Man soll letzterem möglichst nichts Redaktionelles überlassen; denn er hat keine nähere Kenntnis vom Objekt, weder im Feld noch im Büro.

Daraus ergeben sich für unsere Felszeichnung folgende Konsequenzen: Im Gegensatz zur herrschenden Praxis, wo die Felslinien erst bei der Reproduktion zugefügt werden, müssen sie, wie bereits oben vermerkt, unbedingt gleich am Autographen herausgegeben werden, trotzdem die Autographenstunde mit mindestens Fr. 20.zu veranschlagen ist (2 Mann und Autograph). Diese Mehrbelastung des Autographen ist nicht so schlimm, wie sie auf den ersten Blick erscheint. Die Felsränder werden punktiert herausgegeben. Diese sind nichts anderes als typische Felslinien, nämlich die der Umrandung! Dazu braucht es nur ganz wenige Ergänzungs-Felslinien in der Felsfläche. Praktisch kommt es darauf hinaus, daß diese äußern und innern Felslinien ineinander verlaufen, nämlich in dié Felslinien überhaupt. Bald sind es Umrißlinien, bald läuft die Umrißlinie in die Fläche hinein, als eine Linie, bald ist 


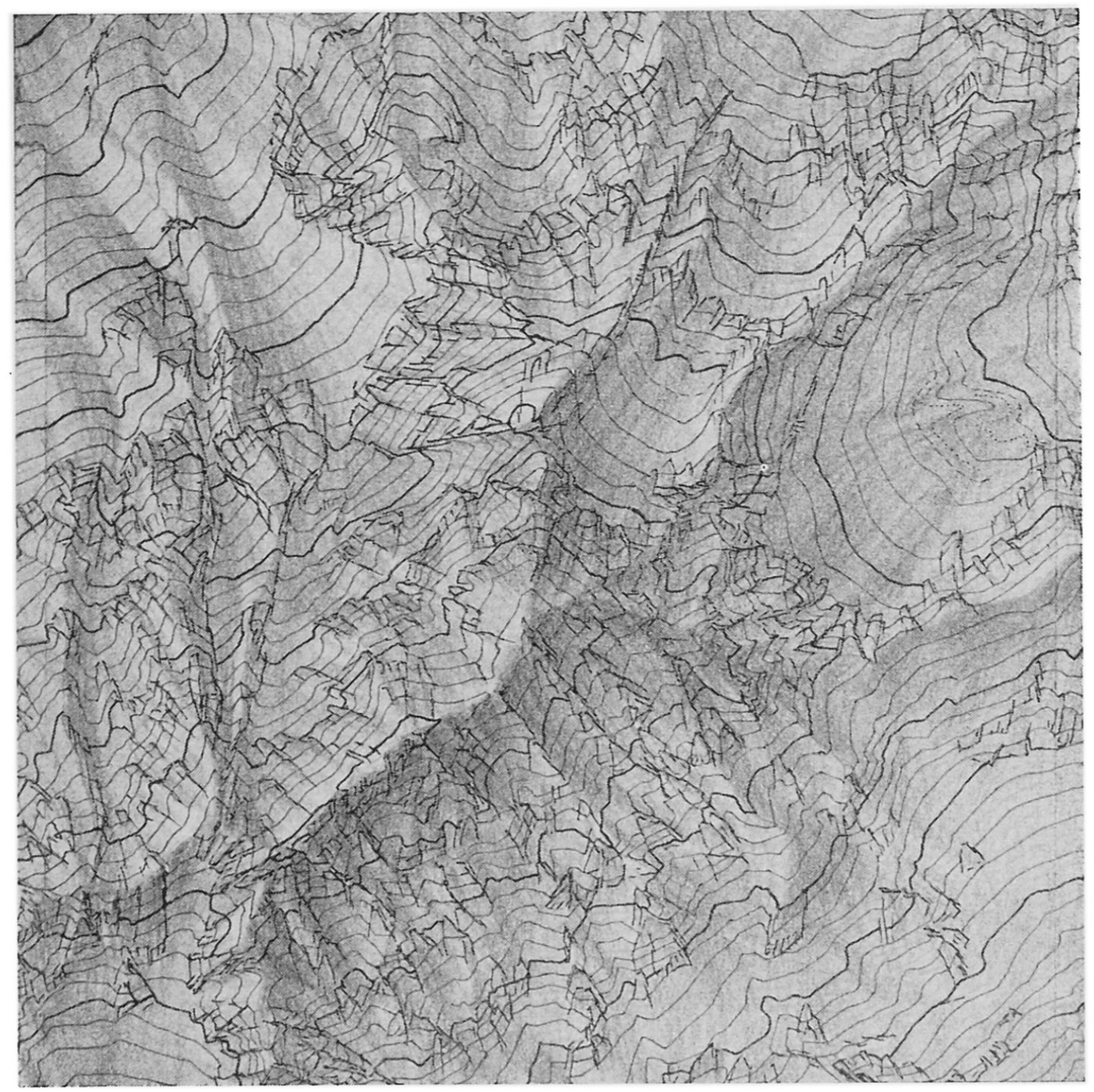

Abb. 13 Ausschnitt aus dem Übersichtsplan Val d'Arpetta, Clochers d'Arpetta. Äquidistanz $20 \mathrm{~m}$. Felskurven, Felslinien und Reliefton. Zeichnung und Bemalung vom Verfasser. Felslinien offen, damit Platz für Eintragungen, was dem Zweck des Übersichtsplanes entspricht. 1:1

sie zusätzlich im Innern, alles je nach der Form der betreffenden Fazette. Daß man damit den Felsen besser gerecht wird, als bloß mit einer summarischen Umrandung, ist klar; besonders in aufgelösten Felsgebieten. Die Felskurven werden vorgängig ganz durchzogen.

Jetzt wird das Blatt ausgezogen; zuerst das Gerippe der Felslinien und als Flächenergänzung das Kurvensystem nach der auflösenden Regel auf $2 \mathrm{~mm}$ Distanz. Hat man zusätzliche Schummerung im Fels nach schiefer Beleuchtung, dann ist die physische Gestaltung der Zeichnung maximal; aber, wie schon gesagt, es geht auch ohne diese.

Dieses ausgezogene Autographenblatt kann jederzeit in photographischer Kopie abgegeben werden, sei es nun an den Reproduktionskartographen, den Zivilingenieur, den Wissenschafter, und keiner wird etwas vermissen, und, was ebenso wichtig ist, keiner findet auf dem Blatt etwas, das ihn an der weiteren Verarbeitung des Blattes hindert oder stört.

Interessant ist nun besonders die neuestens vorgeschriebene einfarbige Reproduktion der Autographenblätter für den Übersichtsplan. Unsere Zeichnungen am KarAtlas haben restlos ergeben, daß die neue Felszeichnungsmethode auch einfarbig die 
besten Resultate ergibt, also Felslinien und Felskurven schwarz. Da muß man sich nun aber doch fragen, ob es nicht besser wäre, zur Negativgravur auf Glas zurückzugehen. Jeder möge sich die rationellen Konsequenzen selber ausmalen. Im föderativen Verfahren ist das allerdings weniger gut durchführbar als im zentralisierten Verfahren; es sei denn, daß eine Kunststoff-Folie die Rolle des Schichtträgers übernehmen könnte. Der reambulierende Topograph müßte sein Blatt nicht mehr ausziehen, resp. bloß seinen Reambulierungsbeitrag. Die Frage ist nun die, ob man am Autographen auch eine fertige Felszeichnung herausgeben könnte; ich glaube ja. Es gäbe natürlich eine spezielle Felszeichnung mit maschinellem Gepräge, bei welcher man vor allem die Variation der Strichdicke in den Felslinien und besonders die für alle Felszeichnungen so wertvollen Haarstriche vermißte. Dies wäre aber für den Übersichtsplan 1:1000 kaum schlimm, im Gegenteil korrektiv, da man nicht übermäßig viel auswerten und in die Zeichnung hineinstopfen kann. Es schadet nichts, wenn allerlei überspitzte An-

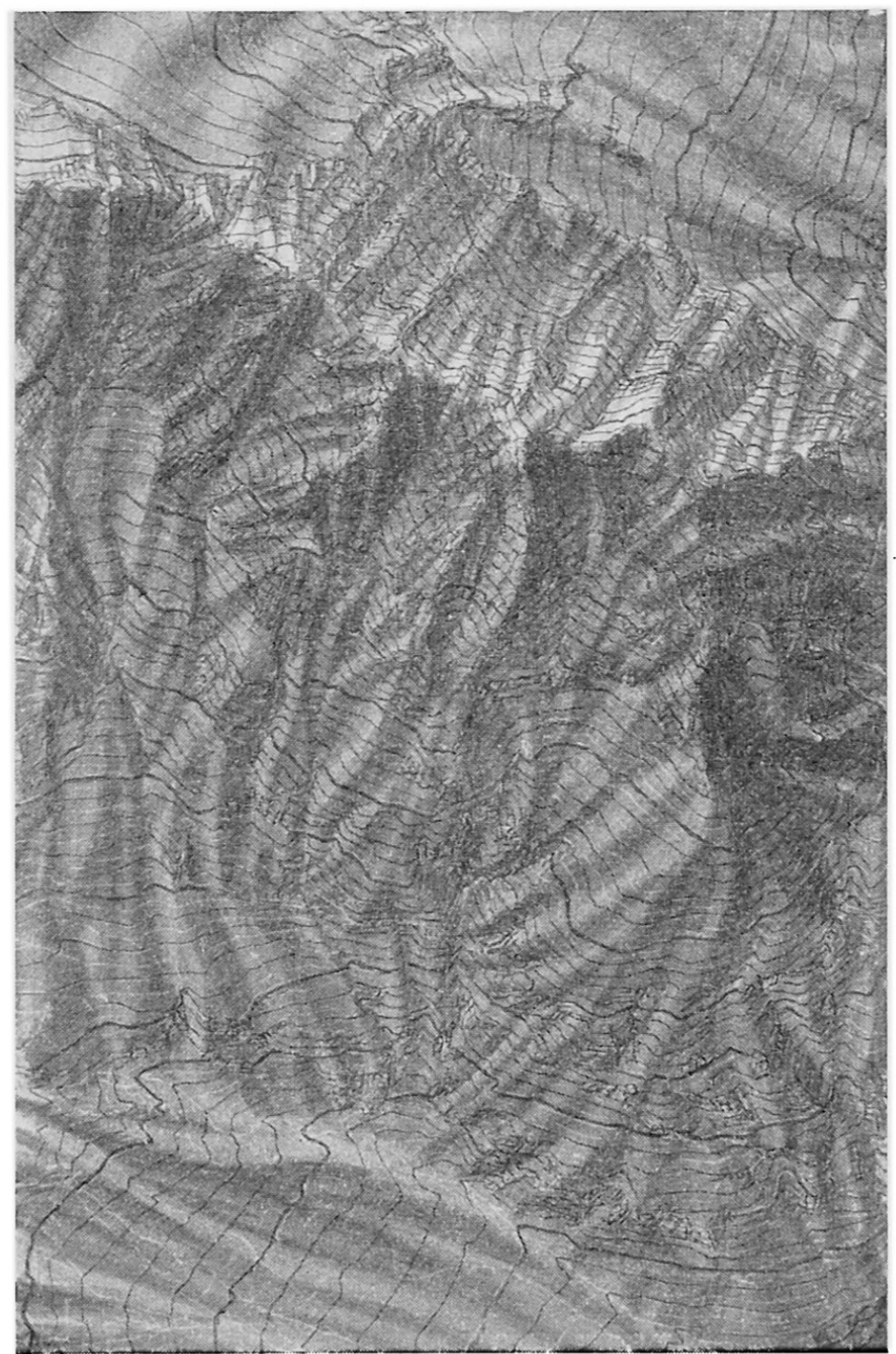

Abb. 14 Ausschnitt aus dem Übersichtsplau Val Ferret. Six Niers. $\ddot{A} q u i d i s t a n z 20 \mathrm{~m}$. Reliefton iu schiefer Beleuchtung, luftperspektivisch abgestimmt. Jede Felszeichnung, sei sie in Schraffen oder photogrammetrisch mit Linien, hat sich in den Tonwerten diesem Reliefton einzupassen. Zeichnung und Bemalung vom Verfasser. Verkleinert

sprüche am Übersichtsplan, der ja ein Grundlagenplan und kein Kartenwerk ist, verschwinden. Die reichliche Erfahrung in der Benützung und weitern Verarbeitung des Übersichtsplanes ${ }^{6}$ zeigte mir immer wieder, daß am Übersichtsplan, beson-

6 Der schweizerische Übersichtsplan $1: 10$ ooo ist durch ähnliches Vorgehen im Ausland präjudiziert. Allen diesen Aufnahmen im Ausland ging in der napoleonischen Aera die französische Katasterverwaltung mit dem Maßstab 1:10000 voran. Die Ausführung dieses 10 ooostels in der Schweiz lehnte sich stark an die Schießkarte 1:10 ooo der Festungsartillerie an, da man bei dieser in diesem Maßstab bereits einige Erfahrung hatte. Diese Festungskarte ist aber kartenmäßig und nicht planmäßig aufgezogen und hat $10 \mathrm{~m}$ Aequidistanz. Obschon ich mit dieser Festungskarte einige Erfahrung besitze, indem ich als Geniesoldat an den Aufnahmen dazu im Feld sowie an der Konstruktion von Schießkarten in verschiedenen Schieß-

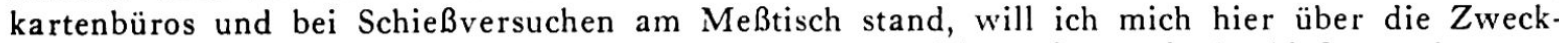
mäßigkeit der topographischen Elemente dieser Karte nicht äußern, glaube bloß erwähnen zu dürfen, daß der Übersichtsplan sich zu stark an diese Festungskarte angelehnt hat. 


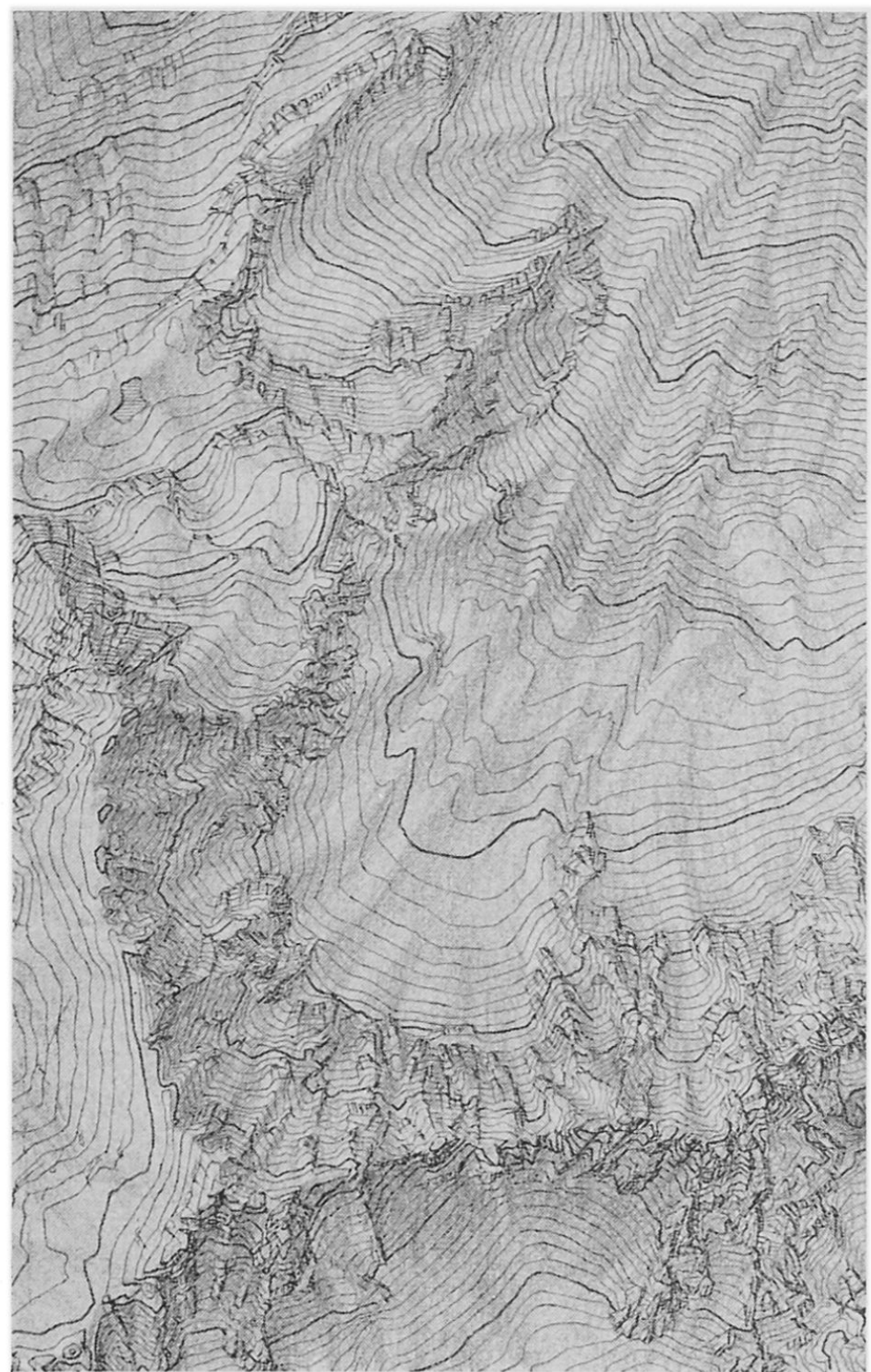

Abb.15 Ausschnitt aus dem Übersichtsplan Val d'Arpetta. Dzennepi. Äquidistanz $10 \mathrm{~m}$. Felskurven, Felslinien und Reliefton. Zeichnung und Bemalung vom Verfasser. Im Gegensatz zu Abb. 13 enges Kurvensystem und engere Felslinienzeichnung, was der Skizze mehr kartographisches Gepräge verleiht. Verkleinert ders aber am Gebirgsübersichtsplan, zu viel ausgewertet wird; vieles braucht man schlechthin überhaupt nicht. $W$ ünschenswert wäre aber eine sorgfältige Preambulierung. Es ist hier aber nicht der Ort, den Übersichtsplan weiterhin $2 \mathrm{u}$ analysieren.

Beleuchtung und Luftperspektive. Alle unsere Zeichnungen bewiesen, daß eine Felsdarstellung mit Kurven und Felslinien durchaus für sich allein bestehen kann, um als Bild von Felspartien wirken zu können (siehe auch Abb. 12). Dasselbe ließ sich bei unseren Abbildungen 1317 feststellen, bevor der $\mathrm{Re}^{-}$ liefton eingelegt wurde. $\mathrm{Zu}$ dem war bis zu einem gewissen Grade auch die Felslinienzeichnung als Träger von Beleuchtung und Luftperspektive benützt worden. Für den vollen physischen Effekt ist aber die Einführung eines besonderen Beleuchtungsträgers unentbehrlich. Dies geschieht so, daß man eine Schummerung einlegt, die als modellierten Schattenton die Fazetten füllt. Sind die Flächen nicht allzu groß, so ist die Verwendung eines Farbstiftes außerordentlich bequem. Bei großen Flächen wird mit dem Pinsel vorgemalt und mit dem Farbstift retouchiert. Zuletzt ist zur Technik dieser neuen photogrammetrischen Methode doch zu erwähnen, daß das bei der alten Schraffenmethode über Luftperspektive Gesagte auch hier gilt (siehe Abb. 14).

Zusammenfassung. Mit Genugtuung ist zu konstatieren, daß wir in der neuen Methode ein Darstellungsverfahren besitzen, bei dem man die einzelnen Operationen getrennt und nicht auf einmal ausführen kann. Alle Operationen sind dadurch viel leichter zu meistern. Zudem kann man einen beträchtlichen Teil davon der Maschine überlassen. Noch ist die Darstellung kein Kinderspiel, aber welche Erleichterung bietet sich gegenüber früher!

Die neue Methode ist natürlich stark vom Maßstab beeinflußt. In großen Maßstäben kommen die einzelnen Komponenten zur vollen Entfaltung. In kleineren werden die Kurven immer mehr von den Felslinien verdrängt, bis schließlich bloß noch die Felslinien übrigbleiben. Schon bei 1:50 000 setzt dieser Prozeß ein, was aber kein 
Nachteil, sondern ein Vorteil ist. Was soll die Felskurve überhaupt in kleinen Maßstäben noch? Wir haben doch an Umrißzeichnung und Reliefton genug, und wo noch Platz bleibt, füllen die Kurven den Raum. Der Reliefton liegt ohnehin von der übrigen Terrainzeichnung her auf dem Blatt. Welch herrliche Einheitlichkeit in den Darstellungsmitteln, die wir da erreichen haben! Kein artfremdes Darstellungsmittel macht sich mehr breit, artfremd wie die Schraffe in einer Kurvenzeichnung war!

\section{E R G E B N I S S E}

Als Hauptresultat der Untersuchung ergibt sich, daß die Felsschraffe im Zeitalter der Photogrammetrie absolut entbehrlich ist, mehr noch, aus allen Plänen und Karten verschwinden sollte. Man wird das aber nicht befolgen, oder nur zögernd, langsam, Schritt für Schritt, was berechtigt ist, da die Ausdruckskraft der Schraffe derart groß und in schwarzer Ausführung derart prägnant erscheint, daß es sehr schwer fällt, darauf zu verzichten. Welche herrliche Sprache redet doch die Dufourkarte, besonders in Kupferabzügen, und was ist ein lithographiertes, wenn auch geschummertes Blatt mit Kurven dagegen! Genau so im Fels. Auch in der Darstellung der Details vermag die neue Methode nie ganz mit der Schraffenmethode Schritt zu halten; es steckt zuviel Maschinenarbeit darin. Dennoch wird sie auf der ganzen Linie siegen. Die Entpersönlichung, welche die Maschine bei Arbeit nach der neuen Methode gebracht hat, ist eben kein Nachteil für die Karte. Man lasse zwei Topographen dasselbe Felsgebiet in Schraffenzeichnung darstellen; sie werden stark variieren. Zwei photogrammetrische Felszeichnungen nach der neuen Methode von zwei verschiedenen Topographen gezeichnet werden aber viel näher beisammen liegen. Die verschiedene künstlerische und wissenschaftliche Auffassung wird sich nicht mehr so stark äußern können. Zudem ist die neue Methode von jedem Vermessungsfachmann ausführbar; die Schraffenmethode dagegen braucht einen begabten Topographen, für einen weniger befähigten auf jeden Fall lange Vorübung. Für nachfolgende Umarbeitungen in andere Maßstäbe oder auch bloßes Umzeichnen im selben Maßstab wird deshalb jeder Praktiker Grundlagen nach der neuen Methode vorziehen. Sie liefert immer eine brauchbare Unterlage, auf der man mit Hilfe der Photographien jederzeit wieder aufbauen oder gar wenn nötig auf Schraffen umzeichnen kann. Die neue Methode ist also die gegebene Methode für den Übersichtsplan 1:10 000, der bekanntlich allen möglichen Situationen dienen soll.

Einst machte der Deutsch-Österreichische-Alpenverein eine Umfrage über unser Thema und legte ein Felsbeispiel einerseits in Schraffen, anderseits in Kurven vor: 100 Anfragen, 76 Antworten. Geographen, Geologen, Kartographen, Künstler und Bergsteiger (alte Gilde) sprachen sich für das Schraffenbild aus. Die Vermesser und die junge Gilde der Bergsteiger votierten für Kurvendarstellung. Ich bin überzeugt, daß mit der hier skizzierten Methode dieses Verhältnis stark beeinflußt werden könnte. Meine Überzeugung geht sogar viel weiter: wie die Terrainschraffe in den Maßstäben bis 1:500 000 praktisch ausgespielt hat, so wird die Felsschraffe auch bald einmal zu den Fossilien zählen. Diese Ansicht gründet sich nicht zuletzt auf Felsdarstellungen, die ich in allen Maßstäben selber gezeichnet habe.

\section{Abbildungen}

1. Die Reproduktion der Abbildungen 1, 5, 6, 7 und 8 erfolgt mit Bewilligung der Eidg. Landestopographie in Wabern vom 10.4. 58.

2. Die Reproduktion der Abbildungen 3, 4 und 9-17 erfolgt mit Bewilligung der Eidg. Vermessungsdirektion in Bern vom 28.3.58.

3. Die Reproduktion der Abbildung 2 erfolgt mit der Bewilligung des Institut Géographique National in Paris vom 4.3.55.

Die Originalzeichnungen zu den Abbildungen $12-17$ hat der Verfasser alle ohne Feldbegehung und ohne Benützung von Photos, rein im Büro auf Grund der Kurven erstellt. Sie sind auch lediglich mit Buchdruck reproduziert worden. Mit Feldbeobachtung, Photos und Negativgravur wären natürlich viel bessere Resultate erreichbar. 


\section{LA REPRÉSENTATION PHOTO-TOPOGRAPHIQUE DU ROCHER}

L'introduction de la stéréophotogrammétrie dans le domaine de la topographie nous a donné une nouvelle méthode graphique pour le dessin du rocher. En lieu et place des hachures, comme jadis, on utilise, grâce au nouveau procédé, des courbes de niveau avec lignes caractéristiques et estompage.

Pour pouvoir juger des nouvelles possibilités photogrammétriques, il est nécessaire de connaître à fond l'ancienne méthode des hachures.

La comparaison des deux méthodes, hachures et courbes de niveau, nous montre que la nouvelle méthode est un peu moins élégante, moins expressive, mais beaucoup plus simple et plus facile à exécuter.

\section{EIN NEUER WELTWIRTSCHAFTSATLAS}

Seit 1957 läßt der bekannte JRO-Verlag in München unter der Herausgeberschaft von Dr. ERNsT Kremling und Prof. Dr. GusTav FochleR-Hauke den JRO-Weltwirtschaftsatlas erscheinen, der innert vier Jahren fertiggestellt werden und zwei Bände umfassen soll. Der Verlag hat sich auf diesem kartographischen Gebiet besonders durch die seit 1954 laufend publizierte «aktuelle JRO-Landkarte * (vgl. Geographica Helvetica 1956, S. 138) bereits wertvolle Erfahrungen erworben und mit Erfolg in einer weiten Leserkreis eingeführt. « Auf Grund eingehender Überlegungen * ist man a zu der Form der Wechselblätter gelangt. Die Wirtschaft fast der ganzen Welt ist heute mehr denn je in einer so schnellen Entwicklung begriffen, daß ein zeitnaher Weltwirtschafts-Atlas, wenn er nicht ständig erneuerungsfähig ist, schon kurz nach seinem Erscheinen veraltet. Nur die 'immerwährende' Form vermittelt dem Benützer daher stets die letzten Erkenntnisse der Wirtschaftsgeographie und und Statistik.» Wenn diese Ansicht auch nur bedingte Richtigkeit besitzt, insofern ältere Daten stets ihren Dokumentations- und Vergleichswert und damit Basisbedeutung behalten, so muß ihr natürlich für die Möglichkeit der laufenden Orientierung zugestimmt werden, und das Vorhaben des Verlages ist in diesem Sinne sehr zu begrüßen. Das Unternehmen ist vorläufig auf 164 Hauptdarstellungen berechnet, wobei der Hauptteil, die ersten 114 Hauptkarten, regionalen bzw. Länderbeschreibungen zufällt. Der Rest soll globalen Übersichtskarten gewidmet werden, von welchen bereits eine größere Zahl (Verbreitung der Haustiere und Viehzuchtprodukte, Stein- und Braunkohle, Erdgasproduzenten, Erdölvorkommen und Erdölförderung, Ölleitungen und Ölausfuhrhäfen, Eisenerzvorkommen und -förderung, Roheisen und Rohstahlerzeugung, Elektrizitätserzeugung, Ühersicht wichtiger Rohstoffe, Gold- und Devisenbestände, Erwerbstätige Bevölkerung, Uran, Atomkraftgewinnung, Weltereignisse im 20. Jahrhundert und eine größere Anzahl von Spezialkarten und statistischen Tabellen, z. B. über Netto-Lebensmittelversorgung je Einwohner, Hauptarten der Bodenbenutzung, Kakao, Tee, Fleischverbrauch, Währungen der Erde, Seeschiffahrtskanäle, Luftverkehr, Welteinkommen) erschienen sind. Schon diese kursorische unvollständige Aufzählung vermag die Konzeption und den Reichtum des dargebotenen Stoffes zu belegen. Die Fülle von einzelnen Darstellungen geht aber nicht minder aus dem länderkundlichen Teil hervor, der bereits Staaten aller Erdteile umfaßt. Die Grundlage der Wirtschaftsstrukturschilderung bilden in der Regel mehrfarbige Verwaltungs-, Siedlungs- und Verkehrskarten mit Höhenschichten (sechs Stufen), denen gleichfalls farbige Volksdichte-, Agrar-, Bergbau- und Industriekarten zugeordnet sind. Für die größern und wichtigeren Länder (Frankreich, bei welchem z. B. auch farbige Darstellungen von Spezialkulturen, Betriebsgrößen und Pachtverhältnissen vorliegen, Australien usw.) sind Sonderdarstellungen geschaffen worden, während kleinere oder weltwirtschaftlich zurücktretende zu Gruppen zusammengefaßt sind (z. B. Österreich-Schweiz, Beneluxländer, Tschechoslowakei-Ungarn, Kamerun-Nigeria-S. Thomé-Spanisch-Guinea, Hinterindien, Ecuador-Peru-Bolivien). Die Maßstäbe liegen zwischen 1:150 000 und 1:15000 000, so daß die Möglichkeit relativ weitgehender Berücksichtigung von Einzelheiten besteht, die mittels Spezialkarten (z. B.Verstaatlichte Betriebe in Österreich, Österreichs Übersee-Güterverkehr, Elektrifizierung der französischen Bahnen, Wertwandlung französischer Wirtschaftsgebiete, Wasserstraßen Großbritanniens, Bodenreform und Entwicklungspläne Italiens,.Bodentypen Ungarns, Neulandgewinnung in Kasachstan, Voltaprojekt in Ghana, Regulierungsplan für den Hwangho usw.) noch wesentlich erweitert wird. Der Atlas beschränkt sich indes nicht nur auf Karten nnd Tabellen. Jedem Land ist auf den Rückseiten der Hauptkarten ein landeskundlicher Text gewidmet, der über Lage, Klima, Pflanzen und Tierwelt, Geschichte, Bevölkerung, Landwirtschaft, Bergbau, Industrie, Verkehrswesen, Staat und Wohlfahrt knapp aber doch ausreichend und unter Beigabe des wichtigsten einschlägigen Schriftums orientiert. Auch die globalen Karten enthalten analoge Informationstexte, so da $\beta$ das Gesamtwerk, einmal vollständig vorliegend, ein ausgezeichnetes landeskundlich-volkswirtschaftliches Lexikon repräsentieren wird. Die bei G. FoCHLER-HAUKE, dem weitgereisten Wirtschaftsgeographen liegende Redaktion gewährleistet größtmögliche Sachlichkeit und Weitsicht der ausgewählten Daten. Schon jetzt darf jedenfalls gesagt werden, daß der JRO-Weltwirtschaftsatlas ein willkommenes Hilfsmittel, ein vorzügllches Vademekum des Wissenschafters wie des Praktikers sein wird.

Redaktion 